ARTICLE

Received 29 Aug 2013 | Accepted 5 Mar 2014 | Published 2 Apr 2014

DOI: $10.1038 /$ ncomms 4555 OPEN

\title{
The Menin-Bach2 axis is critical for regulating CD4 T-cell senescence and cytokine homeostasis
}

\author{
Makoto Kuwahara,2, Junpei Suzuki ${ }^{3}$, Soichi Tofukuji ${ }^{3}$, Takeshi Yamada ${ }^{4}$, Makoto Kanoh ${ }^{4}$, Akira Matsumoto ${ }^{4}$, \\ Saho Maruyama', Kohei Kometani ${ }^{5}$, Tomohiro Kurosaki ${ }^{5,6}$, Osamu Ohara ${ }^{3}$, Toshinori Nakayama ${ }^{7,8}$ \\ \& Masakatsu Yamashita1,2,4,9
}

\begin{abstract}
Although CD4 T-cell senescence plays an important role in immunosenescence, the mechanism behind this process remains unclear. Here we show that T cell-specific Menin deficiency results in the premature senescence of CD4 T cells, which is accompanied by the senescence-associated secretory phenotype after antigenic stimulation and dysregulated cytokine production. Menin is required for the expansion and survival of antigen-stimulated CD4 T cells in vivo and acts by targeting Bach2, which is known to regulate immune homeostasis and cytokine production. Menin binds to the Bach2 locus and controls its expression through maintenance of histone acetylation. Menin binding at the Bach2 locus and the Bach2 expression are decreased in the senescent CD4 T cells. These findings reveal a critical role of the Menin-Bach2 pathway in regulating CD4 T-cell senescence and cytokine homeostasis, thus indicating the involvement of this pathway in the inhibition of immunosenescence.
\end{abstract}

\footnotetext{
${ }^{1}$ Department of Immunology, Graduate School of Medicine, Ehime University, Shitsukawa, Toon, Ehime 791-0295, Japan. ${ }^{2}$ Translational Research Center, Ehime University Hospital, Shitsukawa, Toon, Ehime 791-0295, Japan. ${ }^{3}$ Laboratory of Medical Genomics, Department of Human Genome Research, Kazusa DNA Research Institute, Kisarazu, Chiba 292-0818, Japan. ${ }^{4}$ Department of Infection and Host Defenses, Graduate School of Medicine, Ehime University, Shitsukawa, Toon, Ehime 791-0295, Japan. ${ }^{5}$ Laboratory for Lymphocyte Differentiation, RIKEN Center for Integrative Medical Sciences, Tsurumi-ku, Yokohama 230-0045, Japan. ${ }^{6}$ Department of Lymphocyte Differentiation, WPI Immunology Frontier Research Center, Osaka University, Suita, Osaka 565-0871, Japan. ${ }^{7}$ Department of Immunology, Graduate School of Medicine, Chiba University, Chuo-ku, Chiba 260-8670, Japan. ${ }^{8}$ CREST, Japan Science and Technology Agency, Chuo-ku, Chiba 260-8670, Japan. ${ }^{9}$ PRESTO, Japan Science and Technology Agency, Ehime University, Shitsukawa, Toon, Ehime 791-0295, Japan. Correspondence and requests for materials should be addressed to M.Y. (email: yamamasa@m.ehime-u.ac.jp).
} 
A ge-induced alterations of innate and adaptive immunity are generally viewed as detrimental and designated as 'immunosenescense' ${ }^{1}$. Immunosenescence particularly affects the T-cell compartment and is involved in the agerelated decline of immune functions, which increase the susceptibility of elderly individuals to infectious diseases and certain cancers ${ }^{2,3}$. Further, immunosenescence induces a proinflammatory state and increases the susceptibility to autoimmune diseases such as rheumatoid arthritis ${ }^{4,5}$. Evidence indicates that a prominent effect of ageing on immunity is reduced humoral responses, and that ageing is accompanied by alterations of the $\mathrm{CD} 4 \mathrm{~T}$-cell immunity ${ }^{6,7}$. Therefore, understanding immunosenescence requires knowledge of the age-associated alterations of CD4 T-cell functions and induction of cellular senescence.

CD4 T-cell senescence represents a subset of cellular senescence, which is characterized by irreversible proliferation arrest caused by oxidative stress, reactive oxygen species, oncogene activity or the inactivation of tumour suppressor genes $^{8,9}$. These factors contribute to tumour suppression, wound healing and ageing ${ }^{9}$. Senescent cells can significantly harm the tissue microenvironment through the acquisition of a senescenceassociated secretory phenotype (SASP), which is characterized by a striking increase in the secretion of pro-inflammatory cytokines, chemokines, matrix remodelling factors and pro-angiogenic factors $^{10,11}$. These factors deleteriously alter tissue homeostasis, leading to chronic inflammation and cancer ${ }^{8,10,12,13}$. Therefore, cellular senescence may contribute to a component of ageassociated inflammatory responses called 'inflammaging' ${ }^{14}$.

Certain germinal mutations of MEN1, which encodes MENIN, cause multiple endocrine neoplasia type 1 (ref. 15), which is an autosomal-dominant syndrome characterized by concurrent parathyroid adenomas, gastroenteropancreatic tumours and several other tumour types. Menin does not share significant amino-acid sequence similarity with other proteins, but it possesses nuclear localization signals within its $\mathrm{C}$ terminus ${ }^{16}$. Menin interacts with H3K4 methyltransferases, including mixedlineage leukaemia 1 (MLL1) ${ }^{17}$, and it is an oncogenic cofactor for MLL-associated leukemogenesis ${ }^{18,19}$. Also Menin is known to associate with a trithorax family histone methyltransferase complex and contributes to the DNA damage response and cell cycle regulation ${ }^{20,21}$. Paradoxically, Menin recruits polycomb repressive complex 2 (PRC2) at the pleiotrophin (PTN) locus, and its loss abrogates the binding of the PRC2 components enhancer of zeste homologue 2 (EZH2) and SUZ12 to PTN in lung adenocarcinoma cells ${ }^{22}$. Further, Menin associates with the JunD proto-oncogene product (JUND), nuclear factor of kappa light poly peptide gene enhancer in $\mathrm{B}$ cells 1 (NF- $\kappa \mathrm{B})$, peroxisome proliferator-activated receptor gamma (PPAR- $\gamma$ ), SMAD family member 3 (SMAD3) and $\beta$-catenin, indicating its involvement in transcriptional activation and repression ${ }^{23,24}$.

Bach2 (BTB and Cap'n'collar (CNC) homology 1; basic leucine zipper transcription factor 2) belongs to the CNC gene family ${ }^{25}$. B cells preferentially express Bach2, which is critical for somatic hypermutation and class-switch recombination ${ }^{26,27}$, and are involved in the IgG1 memory B-cell formation ${ }^{28}$. Bach2 also participates in T-cell-mediated immune responses ${ }^{29,30}$, regulates $\mathrm{T}_{\text {reg }}$-mediated immune homeostasis and suppresses multiple CD4 T-cell effector programmes ${ }^{29}$. Bach2-deficient animals suffer from lethal lung and small intestinal inflammation. Bach2 is required for maintaining naive $\mathrm{CD} 4 \mathrm{~T}$ cells by suppressing the effector memory-related gene expression ${ }^{30}$. Bach2 deficiency in $\mathrm{CD} 4 \mathrm{~T}$ cells reduces the naive $\mathrm{CD} 4 \mathrm{~T}$-cell numbers and enhances the effector memory $\mathrm{T}$ cells, particularly $\mathrm{T}_{\mathrm{H}} 2$ type. Furthermore, polymorphisms in $B A C H 2$ are associated with multiple inflammatory diseases ${ }^{31-33}$. More recently, the involvement of
Bach2 in memory CD8 T-cell formation has been reported ${ }^{34}$. These findings establish Bach2 as a key regulator of T-cellmediated immune homeostasis.

In this study, we show that T-cell-specific Menin deficiency induces premature $\mathrm{CD} 4 \mathrm{~T}$-cell senescence, which is accompanied by SASP after antigenic stimulation. Furthermore, Meninknockout (KO) naive CD4 T cells exhibited a dysregulated production of cytokines. We identify Bach2 as a direct target of Menin that regulates senescence and cytokine production. ChIP sequencing revealed that Menin binds to the Bach2 locus and controls Bach2 expression through the maintenance of histone acetylation. A decreased Menin binding and the Bach2 expression were detected in the senescent $\mathrm{CD} 4 \mathrm{~T}$ cells. These findings define a critical role of the Menin-Bach2 pathway in regulating CD4 T-cell-mediated immune homeostasis.

\section{Results}

Menin deficiency induces CD4 T-cell senescence. To determine Menin's role in CD4 T-cell functions, we crossed Menin flox/flox mice with CD4-Cre transgenic (TG) mice. As previously reported, the T-cell numbers were moderately decreased in the spleen and mesenteric lymph node of Menin flox/flox x CD4-Cre TG (Menin KO) mice (Supplementary Fig. 1a) $)^{35}$. Menin KO naive CD4 T cells expressed wild-type (WT) levels of interleukin (Il)-2 mRNA after T-cell receptor (TCR) stimulation (Supplementary Fig. 1b). Il-4 and interferon (Ifn) $\gamma$ mRNAs were not detected in either WT or Menin KO naive CD4 T cells (Supplementary Fig. 1b). The early-phase cell division (days 1-3) induced by TCR stimulation was enhanced in the Menin KO naive $\mathrm{CD} 4 \mathrm{~T}$ cells (Supplementary Fig. 1c).

First, we assessed the role of Menin in CD4 T-cell growth in vitro. Although Menin KO naive $\mathrm{CD} 4 \mathrm{~T}$ cells normally expanded after stimulation, the growth rate was reduced after day 4, even in the presence of exogenous IL-2 (Fig. 1a). To assess the effects of Menin deficiency on the cell cycle, we measured the percentage of replicating cells after incubation with 5-ethynyl-2' deoxyuridine (EdU). While approximately $50 \%$ of the WT CD4 T cells were EdU positive, indicating replication (S phase), only approximately $30 \%$ of Menin KO CD4 T cells were positive 7 days after the initial TCR stimulation (Fig. 1b). A reduced number of EdU-positive cells was also detected in the Menin KO CD4 T-cell cultures on days 3 and 5 (Supplementary Fig. 2a). Increased expression of cyclin-dependent kinase (CDK) inhibitors, Cdkn1a, Cdkn2a and Cdkn2b, was detected in the Menin KO CD4 T cells (Fig. 1c). Cell death was increased in the Menin KO CD4 T-cell cultures (Annexin V positive: approximately 40\%) compared with that in WT cultures (Annexin V positive: approximately 20\%) (Fig. 1d). Increased cell death was detected in the Menin KO CD4 T-cell cultures starting 3 days after the initial stimulation (Supplementary Fig. 2b). There was also increased expression of the mRNA encoding pro-apoptotic factors, such as p19 arf, TP53 apoptosis effector (Perp) and phorbol-12-myristate-13-acetate-induced protein 1 (Pmaip1) (Fig. 1e), indicating that the reduced number of Menin KO $\mathrm{CD} 4 \mathrm{~T}$ cells after day 4 was caused by reduced cell cycle progression and an increased rate of cell death.

Early cell cycle arrest accompanied by the increased expression of Cdk inhibitors is a hallmark of cellular senescence ${ }^{8}$, suggesting that premature senescence is induced after TCR stimulation of Menin KO CD4 T cells. Although the Menin-deficient naive CD4 $\mathrm{T}$ cells did not express a senescence-associated $\beta$-galactosidase (SA $\beta$-Gal) activity, a maker of cellular senescence, the proportion of SA $\beta$-Gal-positive cells was increased in the Menin KO CD4 T-cell cultures 3 days after TCR-mediated stimulation (Supplementary Fig. 2c). A strong expression of the SA $\beta$-Gal 


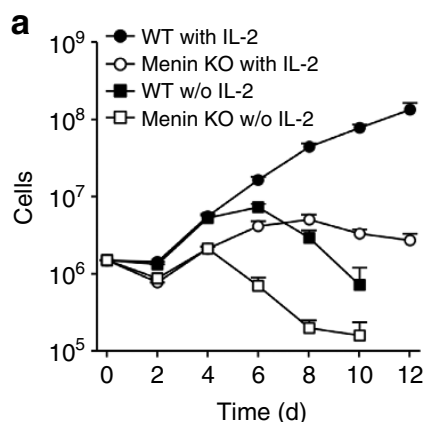

d

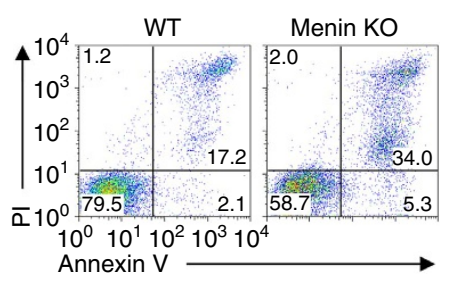

b

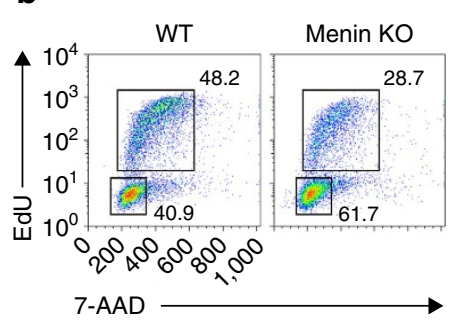

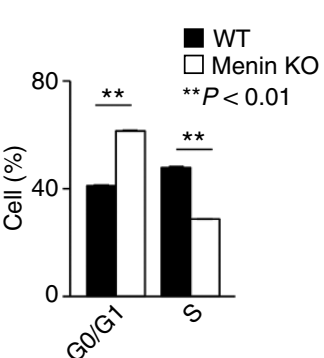

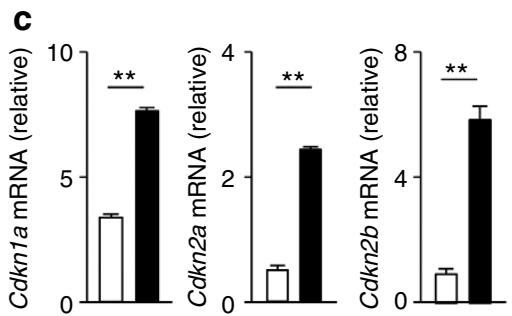

$\square$ WT $\quad$ Menin KO

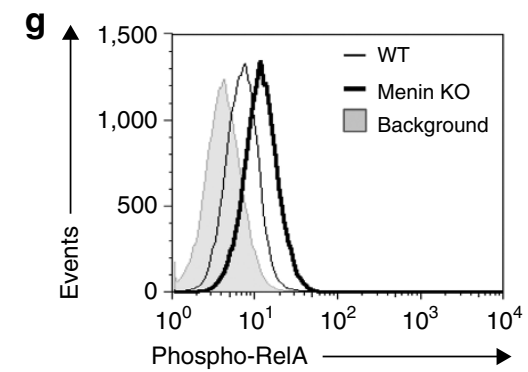

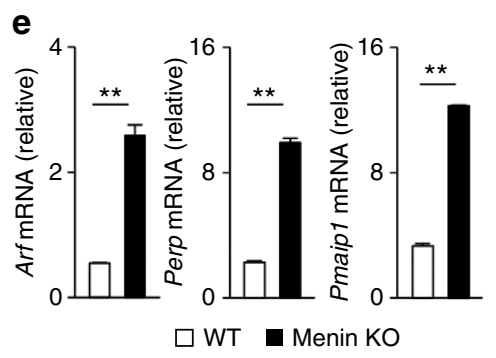

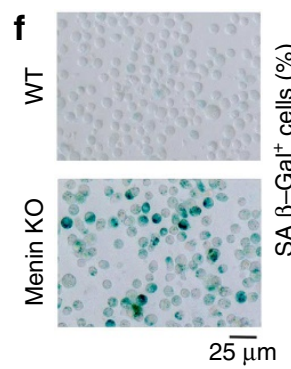

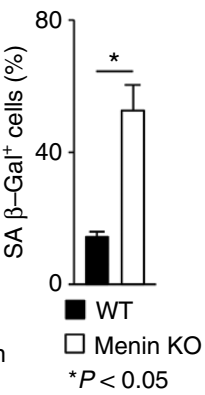

h

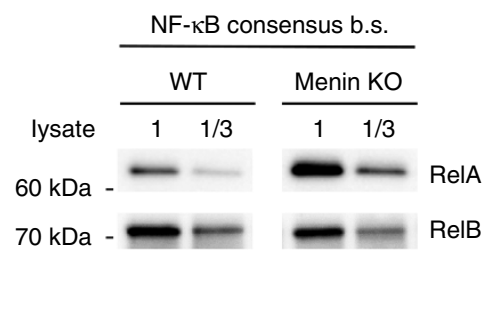

Figure 1 | Menin deficiency induces premature CD4 T-cell senescence. (a) The TCR-induced proliferation of the Menin KO naive CD4 T cells in the presence or absence of IL-2. The average of three independent cultures and s.d. are shown. (b) The results of the cell cycle analysis of CD4 T cells from the Menin KO and WT mice cultured under IL-2 conditions for 7 days. The percentages of G0/G1- and S-phase cells in three independent cultures are shown (right). (c) The results of the quantitative reverse transcriptase polymerase chain reaction (RT-PCR) analysis of mRNA encoding Cdk inhibitors (vertical axes) in the WT and Menin KO TH cells. The results represent the expression levels relative to Hprt with the s.d. (d) The results of the cell death analysis of CD4 T cells from the WT and Menin KO mice on day 7. The percentages of annexin V-positive cells in three independent cultures are shown (right). (e) The results of the quantitative RT-PCR analysis of mRNAs encoding pro-apoptotic proteins (vertical axes) in the WT and Menin KO TH cells, with the s.d. The results are presented relative to the mRNA expression of Hprt with the s.d. (f) SA $\beta$-galactosidase (SA $\beta$-Gal) staining of the $T_{H}$ cells from the Menin KO and WT control mice cultured under IL-2-conditions for 7 days (left). The percentages of SA $\beta$-Gal-positive cells in three independent cultures with the s.d. are shown (right). (g) The phosphorylation of RelA (Ser534) in the WT and Menin KO $T_{H}$ cells cultured for 7 days. (h) Assay of the binding of RelA and RelB to an NF- $\kappa B$-binding consensus motif in the WT and Menin KO $\mathrm{T}_{\mathrm{H}}$ cells cultured under IL-2 conditions for 7 days. Four (panel $\mathbf{c}$ and $\mathbf{e}$ ) and three (panels $\mathbf{a}, \mathbf{b}, \mathbf{d}, \mathbf{f}, \mathbf{g}$ and $\mathbf{h}$ ) independent experiments were performed. ${ }^{\star} P<0.01,{ }^{\star \star} P<0.01$ (Student's $t$-test).

activity was detected in the Menin KO effector $\mathrm{CD} 4 \mathrm{~T}\left(\mathrm{~T}_{\mathrm{H}}\right)$ cells on day 7 (Fig. 1f). NF- $\mathrm{KB}$ signalling induces cellular senescence ${ }^{36-38}$, and the transcription factor NF- $\kappa B$ subunit p 65 (RelA) accumulates on the chromatin of senescent cells ${ }^{39}$. In the Menin KO $\mathrm{T}_{\mathrm{H}}$ cells, enhanced phosphorylation of RelA (pSer534) was detected (Fig. 1g) and its DNA-binding activity increased; however, that of the RelB did not increase (Fig. 1h), thus indicating that the Menin KO CD4 T cells more rapidly senesced after receiving TCR stimulation than did the WT control cells.

The role of Menin in CD4 T response in vivo. To investigate the role of Menin in CD4 T-cell expansion and survival in vivo, we monitored antigen-specific CD4 T cells following immunization in an adoptive transfer model. Naive BALB/c mice were transplanted with the Menin KO or WT naïve CD4 T cells and immunized with ovalbumin (OVA) plus lipopolysaccharide (LPS). After 4 days, the number of transplanted Menin KO cells was approximately $25 \%$ of that of the WT cells, and the number decreased further on day 7 , and the cells did not accumulate in the spleen during the memory phase (day 50) (Fig. 2a).

To further examine the role of Menin in the survival of CD4 T cells after antigenic stimulation, in vitro-activated effector CD4 T cells from WT (CD45.1) and Menin KO (CD45.2) mice were mixed 1:1 and adoptively transferred into naive TCR- $\beta \delta$ KO mice. As shown in Fig. 2b, significant reductions of CD45.2positive Menin KO $\mathrm{CD} 4 \mathrm{~T}$ cells were detected in all the tested tissues (the spleen, liver, lung and PBMCs) 4 weeks after the cell transfer.

Next, we investigated the airway inflammation in Menin KO mice using an in vivo model. WT and Menin KO mice were immunized with OVA containing alum and then were challenged with inhaled OVA. This decreased the inflammatory cell infiltration in the bronchioalveolar lavage (BAL) fluid of the OVA-immunized Menin KO mice compared with that in WT mice (Fig. 2c). The number of mononuclear cells infiltrating the peribronchiolar regions of the lungs in Menin KO mice also decreased (Fig. 2d). 

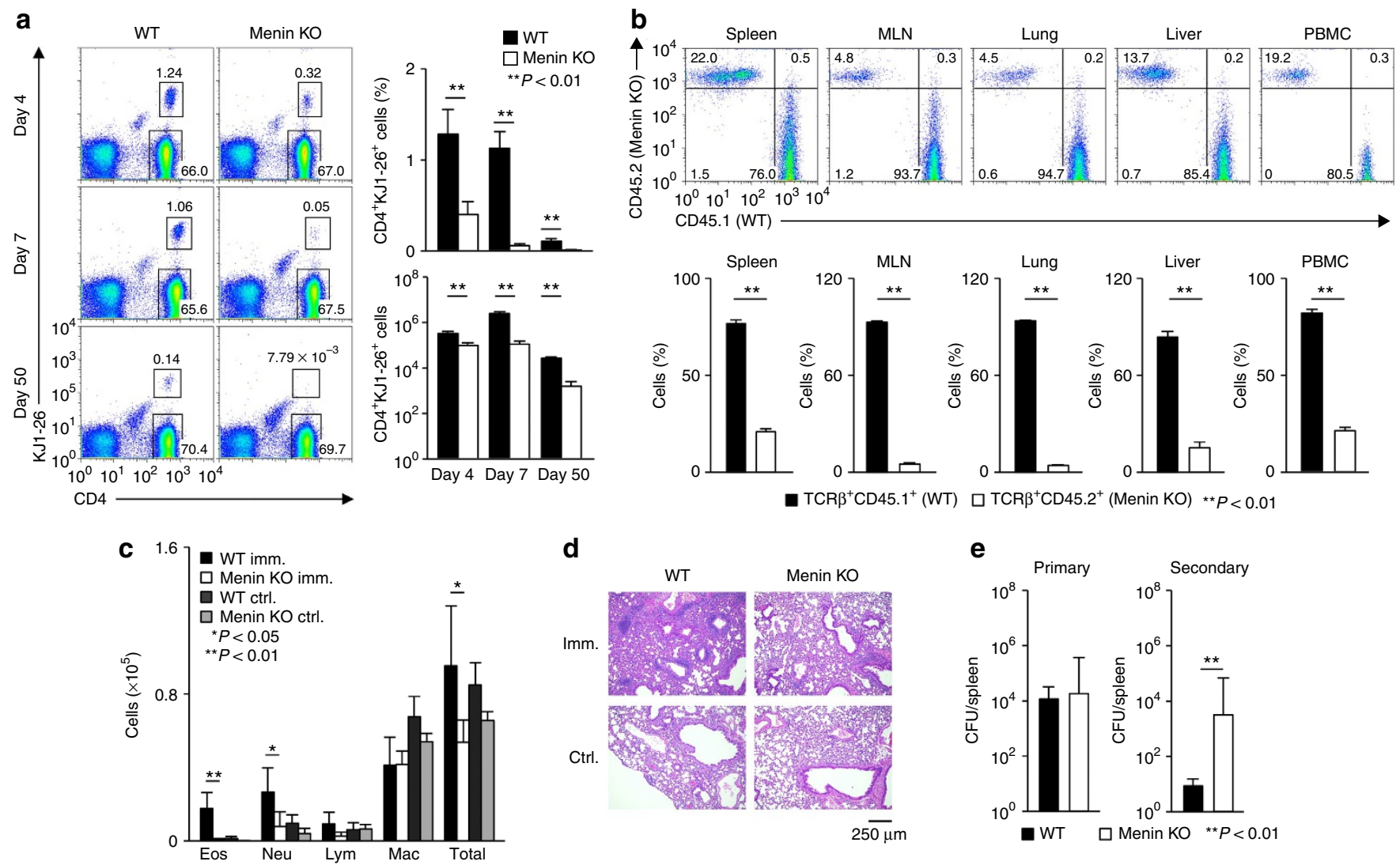

Figure 2 | Menin is required for the expansion and survival of antigen-stimulated CD4 T cells in vivo. (a) CD4 T cells from the spleens of the WT or Menin KO mice with a DO11.10 TG background were transferred into syngenic BALB/c mice, and the mice were then immunized with OVA (100 $\mu g$ ) plus LPS $(10 \mu \mathrm{g})(n=5)$. The transferred OVA-TCR (KJ-1)-positive cells were monitored using FACS on the indicated days. The percentages (upper) and numbers (lower) of KJ1-positive cells are indicated (right). (b) The WT (CD45.1) and Menin KO (CD45.2) TH cells were mixed 1:1 and transferred into TCR- $\beta \delta$ KO mice $(n=5)$. Representative staining patterns gated on TCR- $\beta$-positive cells (upper) and the percentage (lower) of CD45.1 and CD45.2 cells 4 weeks after transfer are indicated. (c) Quantification of eosinophils (Eos), neutrophils (Neu), lymphocytes (Lym), macrophages (Mac) and total cells in the bronchioalveolar (BAL)-fluid from the WT and Menin KO mice ( $n=5$ per group) that were not immunized (ctrl.) or immunized with OVA plus alum (imm.). (d) The microscopic findings of the lungs from mice as in c, fixed and stained with haematoxylin and eosin. (e) The effects of Menin deficiency on L. monocytogenes (LM) infection. The bacterial burden in the spleen was determined 3 days after the primary $(n=6)$ and secondary $(n=8)$ infections, respectively. The data represent the findings of three $(\mathbf{a}, \mathbf{b})$ or two $(\mathbf{c}, \mathbf{d}$ and $\mathbf{e})$ independent experiments. ${ }^{\star} P<0.05,{ }^{\star \star} P<0.01$ (Student's $t$-test).

Finally, we assessed the impact of Menin deficiency on the host defenses against Listeria monocytogenes (Lm) infection. The bacterial burden in the spleens of the Menin KO mice 3 days after primary infection was comparable to that observed in the WT mice (Fig. 2e, left panel). However, the bacterial burden was significantly higher in the Menin KO mice than in the WT mice after secondary Lm infection (recall response) (Fig. 2e, right panel). These results indicate that the expansion, survival and function of antigen-stimulated CD4 T cells in vivo require Menin.

Menin-deficient CD4 $\mathrm{T}$ cells exhibit the SASP phenotype. Decreased cell-surface expression of CD27 and CD62L and increased expression of cytotoxic T-lymphocyte-associated protein 4 (CTLA-4) are characteristics of exhausted and senescent $\mathrm{T}$ cells $\mathrm{s}^{40-42}$. The numbers of CD27 $7^{\text {low }} / \mathrm{CD} 62 \mathrm{~L}^{\text {low }}$ and CD27 $7^{\text {high }}$ / CD62L ${ }^{\text {low }}$ cells were markedly increased in the Menin KO CD4 T-cell cultures compared with those observed in the WT-cell cultures (Fig. 3a and Supplementary Fig. 3a). In addition, moderate upregulation of CTLA-4 was detected in the Menin KO $T_{H}$ cells, the level of which was elevated following immobilized anti-TCR- $\beta$ mAb restimulation (Supplementary Fig. 3b).

The SASP phenotype is characterized by the secretion of proinflammatory factors, including IL-6, IL-8 and pro-inflammatory chemokines $^{10,11}$, and senescent $\mathrm{CD} 4 \mathrm{~T}$ cells produce a high level of osteopontin $(\mathrm{OPN})^{43}$. We found that the number of IL-6- and OPN-producing cells markedly increased in the Menin KO CD4 T-cell cultures, while the number of IL-2-producing cells was marginally decreased (Fig. 3b). Increased production of IL-6 and OPN in the Menin KO T $\mathrm{H}_{\mathrm{H}}$ cells was detected using enzyme-linked immunosorbent assays (ELISAs) (Supplementary Fig. 3c). The production of pro-inflammatory chemokines, chemokine (C-C motif) ligand 3 (CCL-3), CCL-4, CCL-5 and chemokine (C-X-C motif) ligand 2 (CXCL-2), increased in the Menin $\mathrm{KO} \mathrm{T}_{\mathrm{H}}$ cells (Fig. 3c).

Using a DNA microarray analysis, we found that the expression of a series of pro-inflammatory factors was upregulated in the Menin $\mathrm{KO} \mathrm{T}_{\mathrm{H}}$ cells compared with that in the WT cells (Supplementary Data 1). For example, the expression of genes encoding the components of cytotoxic granules (Gzm $a, b$, $c, d, e, f, g$, and $P r f 1)$, which are involved in age-related cardiovascular and pulmonary diseases ${ }^{44}$, markedly increased (Fig. 3d). The augmented expression of pro-inflammatory enzymes such as Adam8, Alox5, Casp1, Ctsd and Ctsh was detected in the Menin $\mathrm{KO} \mathrm{T}_{\mathrm{H}}$ cells (Supplementary Fig. 3d). The increased production of pro-angiogenic factors is another hallmark of SASP in senescent cells ${ }^{10,45}$. The expression of genes encoding pro-angiogenic factors, such as Esm1, Pdgfa, 
a

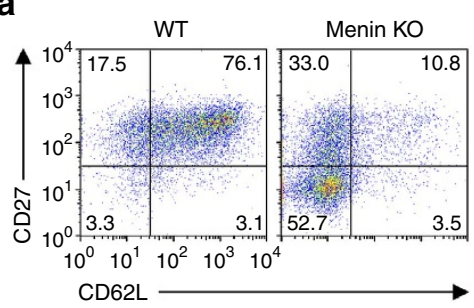

b.
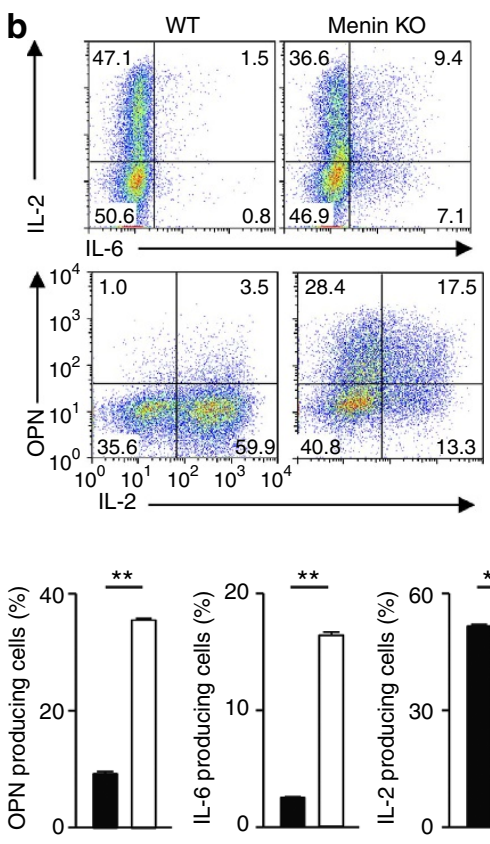

WT $\square$ Menin KO
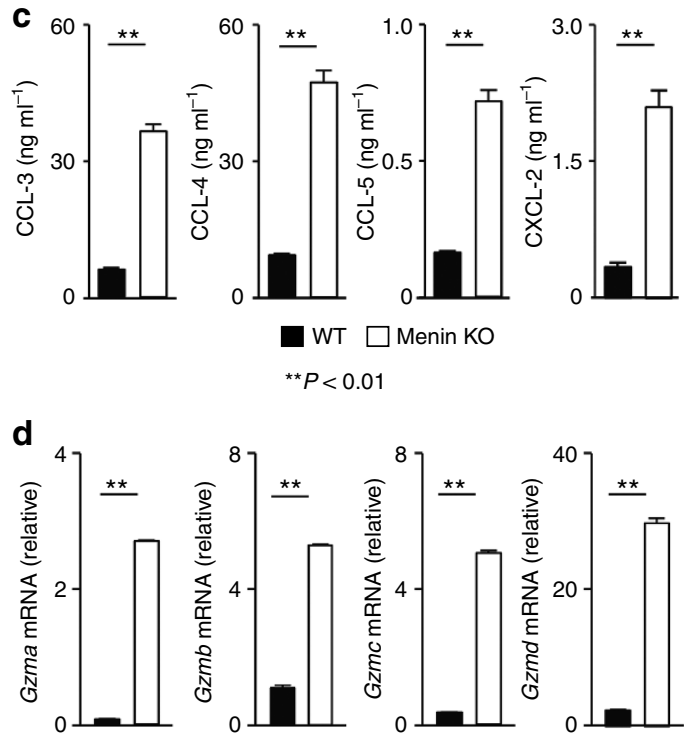

e
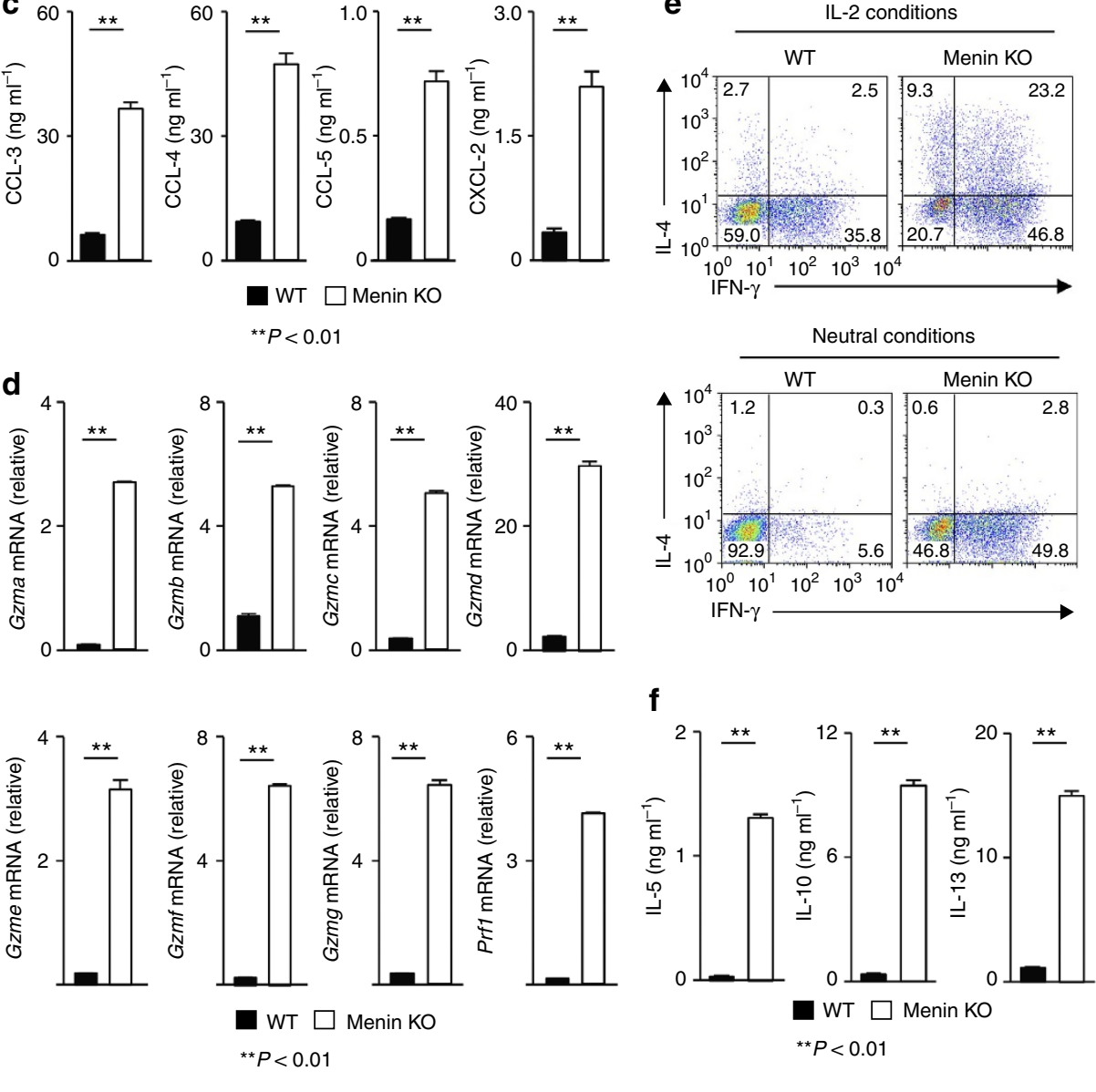

Figure 3 | Menin-deficient effector CD4 T cells exhibit a senescence-associated secretory phenotype. (a) The staining profile of CD62L/CD27 on the cell surface of the WT and Menin KO TH cells. The cell numbers are indicated in each quadrant. (b) The results of the intracellular FACS analysis of IL-2/IL-6, or IL-2/OPN in the WT and Menin KO TH cells. The percentages with s.d. of IL-2-, IL-6- and OPN-producing cells in three independent cultures are shown (lower). (c) The ELISA of chemokines in supernatants of the cells in $\mathbf{b}$. (d) The results of the quantitative RT-PCR analysis of Gzm's and Prf1 in cells in $\mathbf{b}$. The results are presented relative to the expression of Hprt mRNA with the s.d. (e) The results of the intracellular FACS analysis of IL-4 and IFN- $\gamma$ in cells cultured under IL-2- (top) or neutral-conditions (bottom). The numbers of cells are indicated in each quadrant. (f) The ELISA of cytokines in supernatants of the cells cultured under IL- 2 conditions and restimulated with an immobilized anti-TCR- $\beta$ for $16 \mathrm{~h}$. All experiments were performed with $T_{H}$ cells cultured under IL-2 conditions for 5 days. At least three independent experiments (a-f) were performed. ${ }^{\star \star} P<0.01$ (Student's $t$-test).

S100a4 and Vegfc, upregulated in the Menin $\mathrm{KO} \mathrm{T}_{\mathrm{H}}$ cells (Supplementary Fig. 3e). These results strongly suggest that Menin deficiency causes the SASP in the effector CD4 T cells.

Cytokine productions in Menin-deficient CD4 T cells. Changes in the cytokine production profile of senescent CD4 T cells have been previously reported ${ }^{46}$. Next, we analysed the cytokine productions in Menin KO effector CD4 $\mathrm{T}$ cell in vitro. The generation of IFN- $\gamma$ - and IL-4-producing cells was increased in the Menin KO naive CD4 T cell cultured under IL-2 conditions compared with that observed in the WT cells (Fig. 3e upper panel and Supplementary Fig. 4a). Furthermore, IL-4/IFN- $\gamma$-double producers were induced in Menin KO CD4 T-cell cultures (23.2\%) but not in WT cultures (2.5\%) (Fig. 3e upper panel and Supplementary Fig. 4a). Enhanced generation of IFN- $\gamma$ producing cells was detected even in the presence of an antiIFN- $\gamma$ mAb, whereas the generation of IL-4-producing cells was blocked by an IL-4-neutralizing antibody (Fig. 3e lower panel, Supplementary Fig. 4a,b). The production of $\mathrm{T}_{\mathrm{H}}$ 2-related cytokines, such as IL-5, IL-10, and IL-13, was increased in the Menin KO $\mathrm{T}_{\mathrm{H}}$ cells (Fig. 3f), while the IL-9, IL-17A and tumour necrosis factor (TNF)- $\alpha$ production levels remained unaffected under IL-2 conditions (Supplementary Fig. 4c).

To assess the responsiveness of the Menin KO naive CD4 T cells to IL-4 and IL-12, we stimulated naive CD4 T cells with antiTCR- $\beta$ and anti-CD28 Ab in the presence of various doses of IL-4 or IL-12. As we previously reported ${ }^{35}$, the Menin-deficient CD4 T cells normally differentiated into IL-4-producing cells under optimal $\mathrm{T}_{\mathrm{H}} 2$ culture conditions (Supplementary Fig. 4d, right panel). Compared with WT cells, the Menin KO naive CD4 T cells readily differentiated into IL-4-producing cells in response to low concentrations of IL-4 (Supplementary Fig. 4d). Enhanced generation of IFN- $\gamma$-producing cells was detected in the Menin KO CD4 T cells even in the presence of IL-4 (Supplementary Fig. 4d). The IL-12-dependent induction of IFN- $\gamma$-producing cells was augmented in the Menin KO naïve CD4 T-cell cultures, although the Menin-deficient CD4 T cells normally differentiated into IFN- $\gamma$-producing cells under optimal $\mathrm{T}_{\mathrm{H}} 1$ culture conditions (Supplementary Fig. 4e). These results indicate the crucial role of Menin in regulating the IFN- $\gamma$ and $\mathrm{T}_{\mathrm{H}} 2$ cytokine production in CD4 T cells under the condition with limited concentration of IL-4 or IL-12, respectively. 
Bach2 is a downstream target of Menin. Next, we investigated the molecular mechanisms involved in the induction of SASP and cytokine production in the Menin-deficient cells. On the basis of the results of the DNA microarray analysis, we focused on Bach2, which was differentially expressed in the Menin $\mathrm{KO} \mathrm{T}_{\mathrm{H}}$ cells (Supplementary Data 1). The Bach2 mRNA (Fig. 4a, right) and protein levels (Fig. 4b) were significantly reduced in the Menin $\mathrm{KO} \mathrm{T}_{\mathrm{H}}$ cells compared with those in the WT cells. The Bach2 mRNA level in the Menin KO naive CD4 T cells was marginally reduced (Fig. 4a, left). Next, we transduced Bach2 into the Menin KO $\mathrm{T}_{\mathrm{H}}$ cells. The Bach2 protein levels in the Bach2-transduced $\mathrm{T}_{\mathrm{H}}$ cells were comparable to those observed in the WT $\mathrm{T}_{\mathrm{H}}$ cells (Supplementary Fig. 5a). The induction of IL-4- and IFN- $\gamma$ producing cells from the Menin KO naïve CD4 $\mathrm{T}$ cells was normalized by the transduction of Bach2 (Fig. 4c, upper panel). The generation of IL-6-producing cells from the Menin KO naive CD4 T cells was also suppressed by the transduction of Bach2 (Fig. 4c, lower panel). Reduced production of IL-5, IL-10, IL-13 and OPN in the Bach2-transduced Menin $\mathrm{T}_{\mathrm{H}}$ cells was detected using ELISAs (Fig. 4d). The augmentation of CCL-3, CCL-4, CCL-5 and CXCL-2 in the Menin $\mathrm{KO} \mathrm{T}_{\mathrm{H}}$ cells was also normalized by the Bach 2 transduction (Fig. 4e). The transduction of the Menin $\mathrm{KO} \mathrm{T}_{\mathrm{H}}$ cells with Bach2 restored the enhanced
mRNA expression levels of Gzm's and Prf1 (Fig. 4f), Ctla4 (Supplementary Fig. 5b) and the genes that encode proinflammatory enzymes and pro-angiogenic factors (Supplementary Fig. 5c).

We crossed Bach $2^{\text {flox/flox }}$ mice ${ }^{28}$ with CD4-Cre TG mice and generated T-cell-specific Bach2-deficient (Bach2 KO) mice to confirm the roles of Bach2 in CD4 T-cell senescence and cytokine production. Although the number of $\mathrm{T}$ cells was moderately reduced (Supplementary Fig. 6a), a significant number of naive $\mathrm{CD} 4 \mathrm{~T}$ cells remained in the spleens of the Bach2 $\mathrm{KO}$ mice (Supplementary Fig. 6b). The generation of IL-4- and IFN- $\gamma$ producing cells was accelerated, and IL-4/IFN- $\gamma$-double producers were generated in the Bach2 $\mathrm{KO}$ naïve CD4 $\mathrm{T}$ cells cultured under IL-2 conditions (Fig. 5a, upper panel). The augmentation of IL-6-producing cells was observed in Bach2 KO $\mathrm{T}_{\mathrm{H}}$ cells (Fig. 5a, lower). The levels of IL-5, IL-10, IL-13, OPN and pro-inflammatory chemokines were increased in the Bach2 $\mathrm{KO}$ $\mathrm{T}_{\mathrm{H}}$ cells compared with those in WT cells (Fig. 5b). Moreover, the enhanced expression of Gzm's and Prf1 (Fig. 5c), proinflammatory factors (Supplementary Fig. 7a) and pro-angiogenic factors (Supplementary Fig. 7b) was detected in Bach2 KO $\mathrm{T}_{\mathrm{H}}$ cells. The mRNA for Ctla4 was also upregulated in the Bach2 KO $\mathrm{T}_{\mathrm{H}}$ cells (Supplementary Fig. 7c). The expression of the SA $\beta-\mathrm{Gal}$

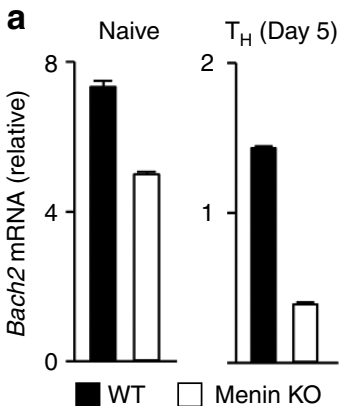

b

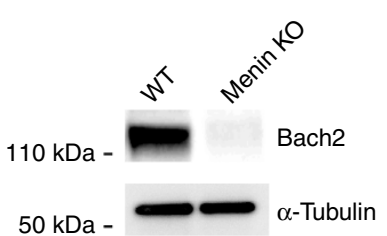

C

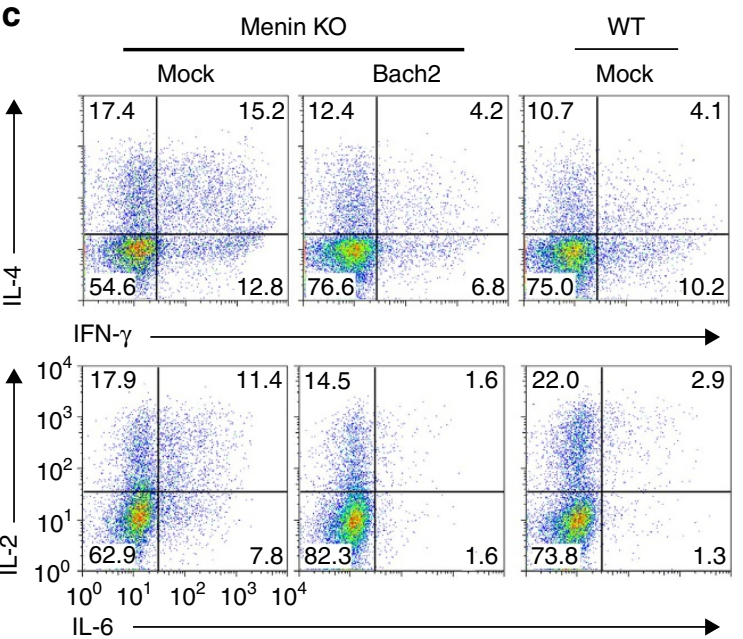

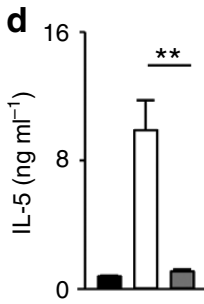
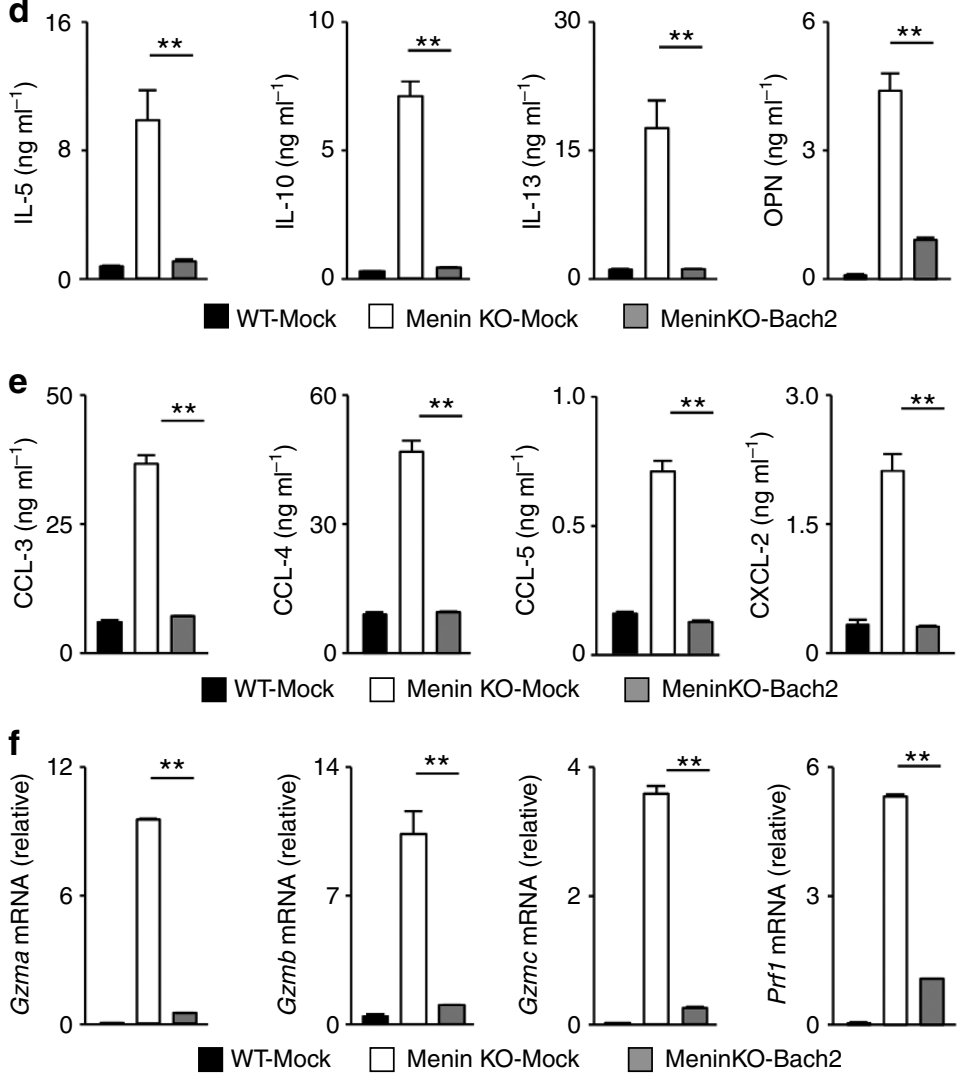

Figure 4 | Enforced expression of Bach2 restores the phenotypes of the Menin KO effector CD4 T cells. (a) The results of the quantitative RT-PCR analysis of mRNA encoding Bach2 in the WT and Menin KO naive CD4 T (right) and $\mathrm{T}_{\mathrm{H}}$ (left). The results are presented relative to the mRNA expression of Hprt with the s.d. (b) The results of the immunoblot analysis of Bach2 in the nuclear fractions of the WT and Menin KO TH cells. (c) The results of the intracellular FACS analysis of IL-4/IFN- $\gamma$ and IL-2/IL-6 in the Menin KO TH cells transduced with an empty vector (Mock) or with Bach2-expressing retroviral vector (Bach2). The naive CD4 T cells cultured under IL-2-conditions were infected with retroviral vectors and cultured for 3 days in the presence of IL-2. (d) The mock- or Bach2-transduced cells in c were purified using an AutoMACS device and restimulated with immobilized anti-TCR- $\beta$ for $16 \mathrm{~h}$.

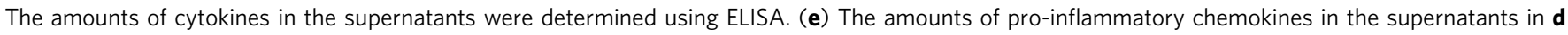
were determined using ELISA. (f) The results of the quantitative RT-PCR analysis of the Gzm's and Prf1 of cells in d. The results are presented relative to the mRNA expression of Hprt with the s.d. All experiments were performed with $T_{H}$ cells cultured under IL-2 conditions for 5 days. Three independent experiments (a-f) were performed. ${ }^{\star \star} P<0.01$ (Student's $t$-test). 

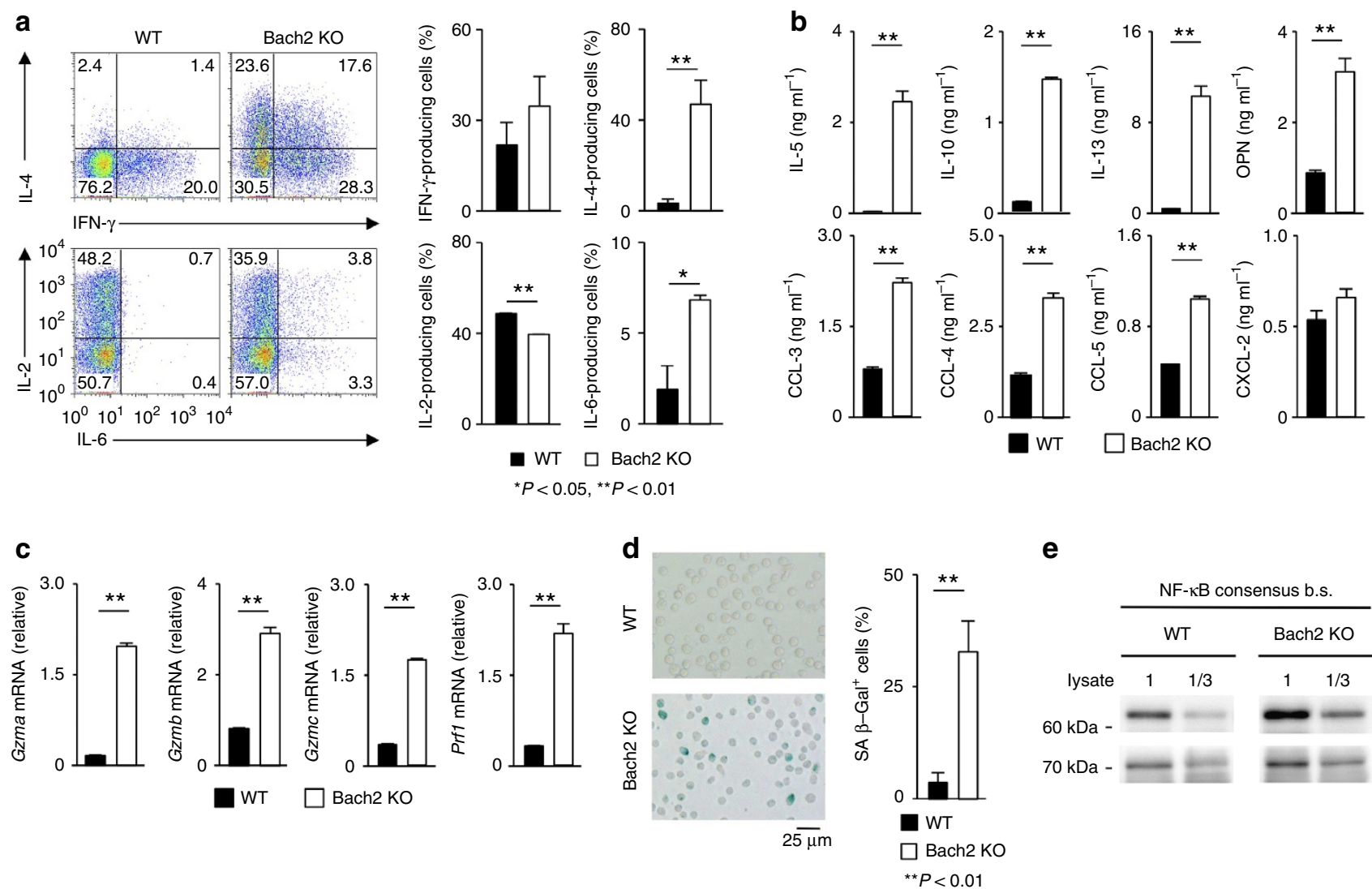

e

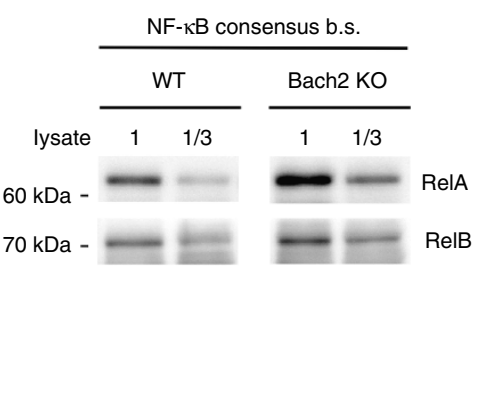

Figure 5 | Early induction of cellular senescence in the Bach2 KO naive CD4 T cells. (a) The results of the intracellular FACS analysis of IL-4/IFN- $\gamma$ and IL-2/IL-6 in the Bach2 KO naive CD4 T cells cultured under IL-2-conditions for 7 days. The percentages with the s.d. of IFN- $\gamma$-, IL-2-, IL-4- and IL-6- producing cells in three independent cultures are shown (right). (b) The ELISA of cytokines and chemokines in the supernatants of the cells in a restimulated with immobilized anti-TCR- $\beta$ for $16 \mathrm{~h}$. (c) The results of the quantitative RT-PCR analysis of the Gzm's and Prf1 of the cells in a. The results are presented relative to the mRNA expression of Hprt with the s.d. (d) SA $\beta$-galactosidase (SA $\beta$-Gal) staining of $T_{H}$ cells obtained from Bach2 KO and WT control mice cultured under IL-2-conditions for 10 days (left). The percentages with the s.d. of SA $\beta$-Gal-positive cells in three independent cultures are shown (right). (e) Assay of the binding of RelA and RelB to an NF- $\mathrm{kB}$-binding consensus motif in the WT and Bach2 KO $\mathrm{T}_{\mathrm{H}}$ cells. The experiment was performed with $T_{H}$ cells cultured under IL-2 conditions for 5 days. Three independent experiments (a-e) were performed. ${ }^{\star} P<0.05,{ }^{\star \star} P<0.01$ (Student's $t$-test).

activity was increased in the Bach2 $\mathrm{KO} \mathrm{T}_{\mathrm{H}}$ cells (Fig. 5d). Furthermore, the DNA-binding activity of RelA was upregulated in the Bach2 $\mathrm{KO} \mathrm{T}_{\mathrm{H}}$ cells (Fig. 5e). These data indicate that the senescent phenotypes of Bach2 $\mathrm{KO} \mathrm{T}_{\mathrm{H}}$ cells are similar to those of Menin KO cells.

Menin maintains histone acetylation at the Bach2 locus. To investigate the molecular mechanism by which Menin controls the Bach2 expression, we assessed changes in the histone modification of Bach2 in the Menin KO $\mathrm{T}_{\mathrm{H}}$ cells using a ChIP-seq analysis. The level of H3K4me3 at Bach2 peaked at the transcriptional start site (Tss) and was slightly decreased in the Menin $\mathrm{KO} \mathrm{T}_{\mathrm{H}}$ compared with that in the WT $\mathrm{T}_{\mathrm{H}}$ cells (Fig. 6a, upper). The level of $\mathrm{H} 3 \mathrm{~K} 27 \mathrm{me} 3$ at the Tss of Bach2 was increased in the Menin KO $\mathrm{T}_{\mathrm{H}}$ cells (Fig. 6a, middle). Furthermore, the level of acetylated (ac) histone $\mathrm{H} 3 \mathrm{~K} 27$ was markedly reduced in the Menin KO $\mathrm{T}_{\mathrm{H}}$ cells (Fig. 6a, lower). We further compared the histone modifications around the Tss of Bach2 between Menin KO and WT CD4 T cells. A schematic representation of the region surrounding the Tss of Bach2 and the location of the amplicons used in ChIP-qPCR are shown in Supplementary Fig. 8a. Decreased levels of H3K4me3 and H3K27ac and an increased level of $\mathrm{H} 3 \mathrm{~K} 27 \mathrm{me} 3$ around the Tss of Bach2 were detected in the Menin $\mathrm{KO}_{\mathrm{H}}$ cells cultured under IL-2 conditions for 5 days (Fig. 6b). Interestingly, a striking reduction in the $\mathrm{H} 3 \mathrm{~K} 27 \mathrm{ac}$ level was detected in the Menin $\mathrm{KO} \mathrm{T}_{\mathrm{H}}$-cell cultured for 3 days, while the H3K4me3 level remained unchanged (Supplementary Fig. 8b). The level of H3K4me3 was also unaffected in the Menin KO naive CD4 T cells (Supplementary Fig. 8b). In addition, we were able to detect only a small reduction in the $\mathrm{H} 3 \mathrm{~K} 27 \mathrm{ac}$ level, as well as upregulation of H3K27me3, in the Menin KO naive CD4 T cells. (Supplementary Fig. 8b). These results suggest that the reduction of $\mathrm{H} 3 \mathrm{~K} 27 \mathrm{ac}$ observed after TCR stimulation has the primary effect of inducing Menin deficiency.

Menin is a component of the KMT2A/2B complex, which is involved in the methylation of histone $\mathrm{H} 3 \mathrm{~K} 4$, an active histone marker ${ }^{17}$. We assessed changes in the binding of KMT2A/2B and PRC2 components at Bach2 in the Menin KO $\mathrm{T}_{\mathrm{H}}$ cells using ChIP-qPCR. As shown in Fig. 6c, the binding of the KMT2A/2B complex, including that with MLL1, Rbbp5 and Wdr5, was decreased in the Menin $\mathrm{KO} \mathrm{T}_{\mathrm{H}}$ cells, whereas the binding of Ezh2 and Suz12 (PRC components) was augmented (Fig. 6c). However, the level of binding of the KMT2A/2B complex with the Bach2 gene was relatively low.

Next, we treated Menin KO $\mathrm{T}_{\mathrm{H}}$ cells with trichostatin A (TSA), an HDAC inhibitor, to determine the role of histone acetylation in maintaining Bach 2 transcription. The Bach 2 expression in the Menin $\mathrm{KO} \mathrm{T}_{\mathrm{H}}$ cells was completely restored by TSA treatment (Fig. 6d). The recruitment of RNA polymerase II and the 
a
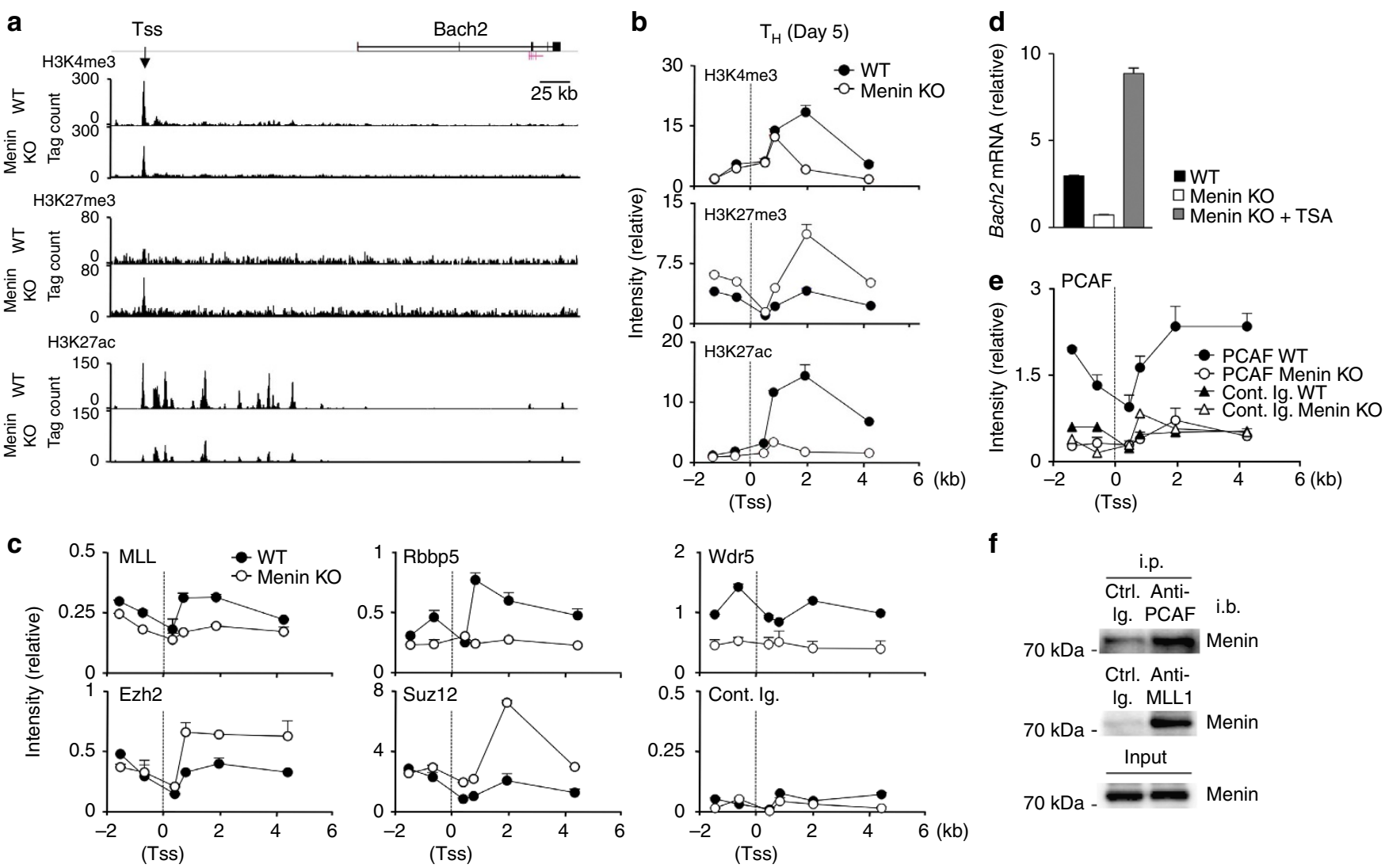

f

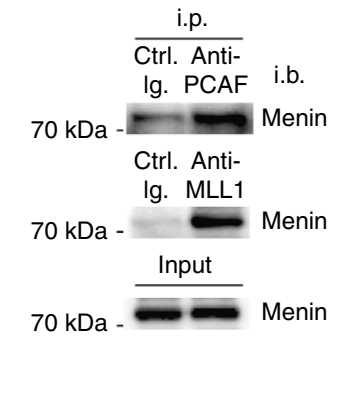

Figure 6 | Menin controls the Bach2 expression by maintaining the histone acetylation at the Bach2 locus. (a) The global patterns of histones, H3K4me3 (upper), H3K27me3 (middle) and H3K27ac (lower) at the Bach2 locus in the WT and Menin KO TH cells cultured under IL-2 conditions for 5 days were determined using ChIP-seq. (b) The results of the ChIP assay with a quantitative PCR analysis of the histone modification (H3K4me3, H3K27me3 and H3K27ac) status around the Tss of the Bach2 locus in the WT and Menin KO TH cells. The relative intensity (input) with the s.d. is shown. (c) ChIP with a quantitative PCR analysis of the binding of HMT2A/B (MLL1, Rbbp5 and Wdr5) and PRC2 (Ezh2 and Suz12) components to the Bach2 Tss region in the Menin KO TH cells. (d) The results of the quantitative RT-PCR analysis of Bach2 mRNA expression in the Menin KO $\mathrm{T}_{\mathrm{H}}$ cells treated with TSA for $24 \mathrm{~h}$. The results are presented relative to the mRNA expression of Hprt mRNA with the s.d. (e) The ChIP data with the results of the quantitative PCR analysis of PCAF binding to the Bach2 Tss region. The relative intensity (/input) with the s.d. is shown.

(f) The association between Menin and PCAF in the $T_{H}$ cells. The nuclear lysates $\left(5 \times 10^{6}\right.$ cells) were precipitated with anti-PCAF mAb or anti-MLL1 $\mathrm{pAb}$, and immunoblotted with anti-Menin pAb. Two (a) and three (b-f) independent experiments were performed. ${ }^{* *} P<0.01$ (Student's $t$-test). All experiments were performed with $T_{H}$ cells cultured under IL-2 conditions for 5 days.

H3K4me3 and H3K27ac status at the Tss of the Bach2 gene locus were also restored by TSA (Supplementary Fig. 8c). We detected enhanced binding of HDAC1 at the Bach2 locus in the Menin KO mice, and the level of which was reduced by TSA treatment (Supplementary Fig. 8d). To identify histone acetyltransferases that are recruited in a Menin-dependent manner, we performed a series of ChIP-qPCR assays and found that the binding of lysine acetyltransferase 2B (PCAF) around the Bach2 Tss was reduced in the Menin KO $\mathrm{T}_{\mathrm{H}}$ cells (Fig. 6e). The binding of other HATs, such as CBP and p300, to the Bach2 Tss in the Menin KO $\mathrm{T}_{\mathrm{H}}$ cells was comparable to that observed in the WT $\mathrm{T}_{\mathrm{H}}$ cells (Supplementary Fig. 8e). In addition, the physical association between PCAF and Menin was detected in the WT $\mathrm{T}_{\mathrm{H}}$ cells (Fig. 6f). These results suggest that the primary result of Menin deficiency is the inability to maintain active histone modification at the Bach2 locus after TCR stimulation.

Menin controls Bach2 expression in the senescent $T_{H}$ cells. To determine whether the expression of Menin and/or Bach2 is reduced in senescent $\mathrm{CD} 4 \mathrm{~T}$ cells, we established a senescent $\mathrm{T}_{\mathrm{H}}\left(\mathrm{sT}_{\mathrm{H}}\right)$ cell induction model (Supplementary Fig. 9a). The $\mathrm{CD} 62 \mathrm{~L}^{\text {low }} / \mathrm{CD} 27^{\text {high }}$ and $\mathrm{CD} 62 \mathrm{~L}^{\text {low }} / \mathrm{CD} 27^{\text {low }}$ populations were increased in the $s \mathrm{~T}_{\mathrm{H}}$ culture in comparison with that observed in the primary effector $\mathrm{CD} 4 \mathrm{~T}$ cells $\left(\mathrm{pT}_{\mathrm{H}}\right)$ culture (Supplementary Fig. 9b). The number of IL-6-, IL-10- and OPN-producing cells was also increased in the $\mathrm{sT}_{\mathrm{H}}$ cultures that observed in the $\mathrm{pT}$ culture (Supplementary Fig. 9c). Therefore, we concluded that $\mathrm{sT}_{\mathrm{H}}$ have senescent $\mathrm{CD} 4 \mathrm{~T}$-cell-like characteristics.

The mRNA and protein expressions of Bach2 in the $\mathrm{sT}_{\mathrm{H}}$ cells were lower than those observed in the $\mathrm{pT}_{\mathrm{H}}$ cells (Fig. 7a,b). In sharp contrast, the expression of Menin in the $\mathrm{sT}_{\mathrm{H}}$ cells was comparable to that observed in the $\mathrm{pT}_{\mathrm{H}}$ cells (Fig. 7a,b). Next, we performed ChIP sequencing using an anti-Menin antibody to compare the binding levels of Menin at the Bach2 locus in the $s \mathrm{~T}_{\mathrm{H}}$ and in $\mathrm{pT}_{\mathrm{H}}$ cells. As shown in Fig. $7 c$, Menin binding was detected around the Tss of the Bach2 gene, spread towards the $3^{\prime}$ sites in the $\mathrm{pT}_{\mathrm{H}}$ cells. The level of Menin binding was decreased in the $\mathrm{sT}_{\mathrm{H}}$ cells, and a reduction in the $\mathrm{H} 3 \mathrm{~K} 4 \mathrm{me} 3$ and $\mathrm{H} 3 \mathrm{~K} 27 \mathrm{ac}$ levels was also detected at the Bach2 in the $\mathrm{sT}_{\mathrm{H}}$ cells (Fig. 7d). The decreased binding of Menin around the Tss at the Bach2 gene locus was confirmed using ChIP-qPCR. A reduction in the binding of RNA polymerase II (PNAPII), PCAF, MLL1, Rbbp5 and Wdr5, as well as an increase in the binding of Suz12 at the Bach2 was also detected in the $s \mathrm{~T}_{\mathrm{H}}$ cells (Fig. 7e). Although the reduced expression of Bach 2 mRNA (Fig. 7d) and the level of $\mathrm{H} 3 \mathrm{~K} 27 \mathrm{ac}$ (Supplementary Fig. 9d) in the $\mathrm{sT}_{\mathrm{H}}$ cells were 
a

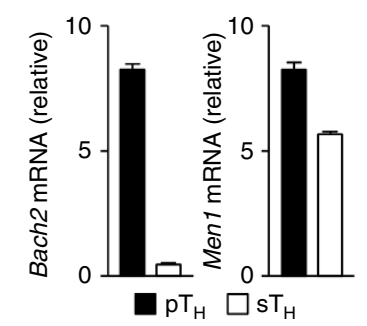

C
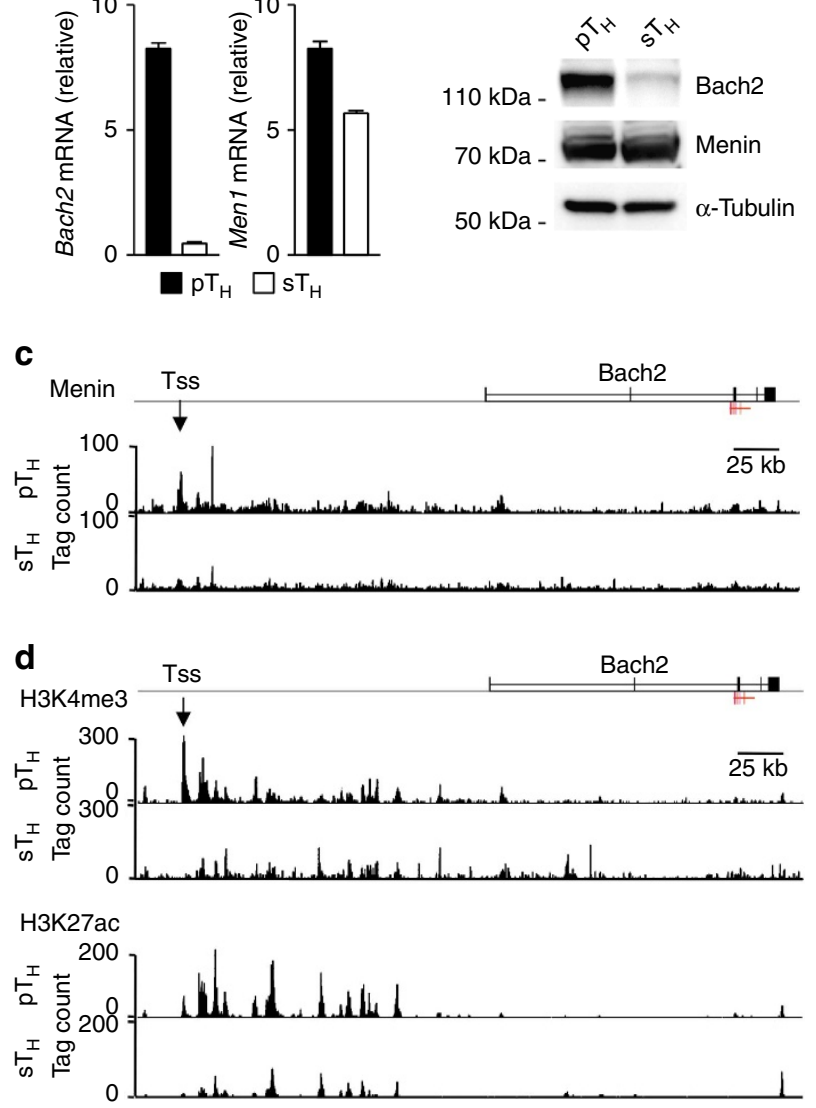

$50 \mathrm{kDa}$ - b
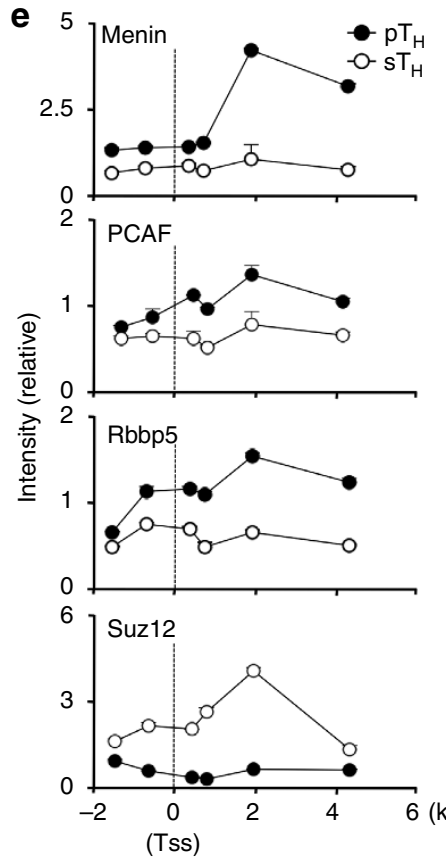

f

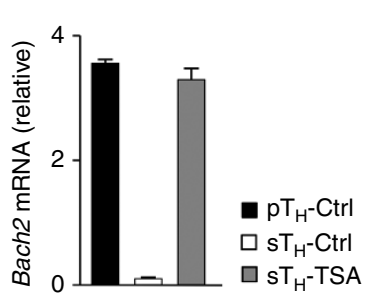

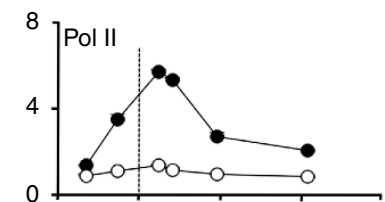
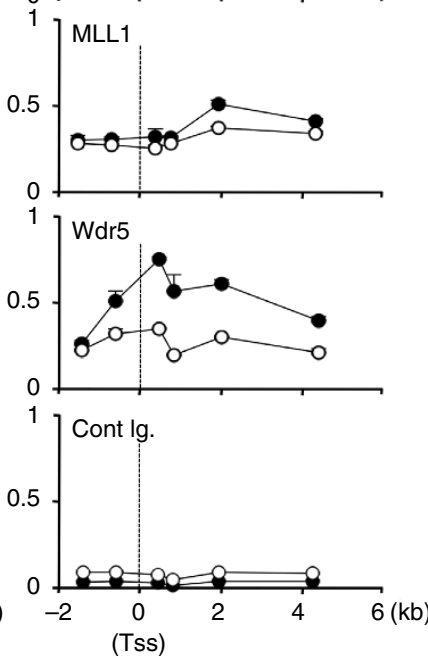

g

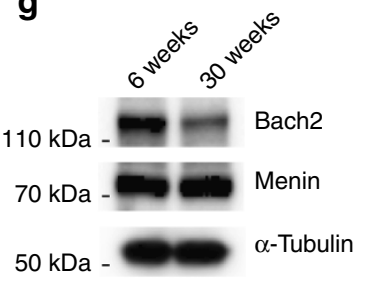

Figure 7 | Menin is involved in the regulation of the Bach2 gene expression in senescent CD4 T cells. (a) A quantitative RT-PCR analysis of the Bach2 and Menin mRNA expression in senescent effector CD4 T ( $\left.\mathrm{sT}_{\mathrm{H}}\right)$ cells. The results are presented relative to the expression of Hprt mRNA with the s.d. (b) An immunoblot analysis of Bach2 and Menin in the nuclear fractions of the primary effector CD4 T (pTH) and sT cells. The $\alpha$-tubulin proteins in the cytosolic fractions were blotted as a loading control. (c) The global patterns of Menin binding at the Bach2 gene locus in the $\mathrm{pT}_{H}$ and $\mathrm{s} \mathrm{T}_{H}$ cells were determined using ChIP sequencing. (d) The global patterns of histones H3K4me3 and H3K27ac at the Bach2 gene locus in the pT $T_{H}$ and $s T_{H}$ cells were determined using ChIP sequencing. (e) ChIP with quantitative PCR analysis of Menin, RNA polymerase II, KMT2A/2B components and Suz12 in the $\mathrm{pT}_{H}$ and $s \mathrm{~T}_{H}$ cells. The relative intensity (input) is shown with the s.d. (f) $A$ quantitative RT-PCR analysis of the Bach2 mRNA expression in the sT cells treated with TSA for $24 \mathrm{~h}$. The results are presented relative to the expression of Hprt mRNA with the s.d. (g) A decreased Bach2 protein expression in the elderly $T_{H}$ cells. Bach2 protein expression in young ( 6 weeks old) and elderly (30 week old) was determined by immunoblotting. Three (a,b,e,f and $\mathbf{g}$ ) and two $(\mathbf{c}, \mathbf{d})$ independent experiments were performed with similar results. ${ }^{\star \star} P<0.01$ (Student's $t$-test).

completely restored by TSA treatment (Fig. 7f), the recruitment of Menin at the Bach2 locus was not increased in the TSA-treated $\mathrm{sT}_{\mathrm{H}}$ cells (Supplementary Fig. 9e). These results indicate that the Menin-dependent maintenance of histone acetylation is required for the proper expression of Bach2, whereas Menin is not essential for Bach2 transcription.

Finally, we compared the expression levels of Bach2 in $\mathrm{T}_{\mathrm{H}}$ cells obtained from young and elderly mice. As shown in Fig. $7 \mathrm{~g}$, the Bach2 expression was lower in the elderly $\mathrm{T}_{\mathrm{H}}$ cells than in the young $\mathrm{T}_{\mathrm{H}}$ cells. In addition, a substantial number of elderly $\mathrm{T}_{\mathrm{H}}$ cells rapidly expressed an SA $\beta$-Gal activity (Supplementary Fig. 10). These results demonstrate a correlation between ageing and the Bach2 expression.

\section{Discussion}

In this study, we demonstrated the critical role that the MeninBach2 pathway plays in regulating CD4 T-cell senescence and cytokine production. Menin KO $\mathrm{T}_{\mathrm{H}}$ cells exhibited phenotypes that are characteristics of cellular senescence, including a reduced growth rate, increased SA $\beta$-Gal activity, prolonged activation of p65/RelA and induction of SASP after TCR stimulation. Furthermore, the generation of IL-4- and IFN- $\gamma$-producing cells was augmented in the Menin KO naive CD4 T cells in particular under IL-2 culture conditions. The transcription repressor Bach2, which inhibits senescence and abnormalities in cytokine production, was identified to be a downstream target of Menin. The transduction of Bach2 into the Menin $\mathrm{KO} \mathrm{T}_{\mathrm{H}}$ cells restored the SASP and cytokine production. In contrast, the Bach2 KO CD4 T cells rapidly expressed a senescent phenotype following TCR stimulation, with an increase in the generation of IL-4- and IFN- $\gamma$-producing cells in particular under IL- 2 culture conditions. The decreased binding of Menin around Tss at the Bach2 gene and a reduction in the Bach2 expression were detected in the artificially induced senescent $\mathrm{T}_{\mathrm{H}}\left(\mathrm{sT}_{\mathrm{H}}\right)$ cells. We thus concluded that the Menin-Bach2 axis represses the transcriptional programme that controls cellular senescence and cytokine production and is involved in immunosenescence.

CD4 $\mathrm{T}$ cells play an essential role in mediating humoral and cellular immune responses. Therefore, the age-associated 
dysfunction of $\mathrm{CD} 4 \mathrm{~T}$ cells has a strong impact on the immune system. Our present study revealed the important role of the Menin-Bach2 pathway in regulating CD4 T-cell homeostasis. $B A C H 2$ polymorphisms are associated with multiple inflammatory diseases in humans ${ }^{31-33}$. Bach2 regulates process of immune homeostasis dependent on $\mathrm{T}_{\text {reg }}$ and multiple effector programmes in CD4 T cells, and the spontaneous onset of lethal lung and small intestinal inflammation occurs in Bach2-null animals ${ }^{29}$. We observed an augmentation of the generation of IFN- $\gamma-$ and $\mathrm{T}_{\mathrm{H}} 2$ cytokine-producing cells in the Bach2 KO naive CD4 T-cell cultures. Taken together, these data suggest that the Menin-Bach2 axis regulates $\mathrm{CD} 4 \mathrm{~T}$-cell immune homeostasis and protects against the onset of multiple inflammatory disorders.

Although Bach2 plays a crucial role in T-cell-mediated immune responses ${ }^{29,30}$, the mechanism regulating its physiological expression is unknown. Our present study reveals the crucial role of Menin in the regulation of Bach2 expression in CD4 T cells. However, Menin may not be essential for Bach2 transcription, because the mRNA expression of Bach2 was only minimally reduced in the Menin-deficient naive CD4 $\mathrm{T}$ cells. Several studies have indicated that Menin is involved in the regulation of gene transcription through histone modifications ${ }^{47-49}$. Menin interacts with histone methyltransferases MLL1 (ref. 17) to regulate transcription. The Menin-MLL histone methyltransferase complex contains other components encoded by Ash $2 l, R b b p 5$ and Wdr5; however, the molecules do not always co-localize with Menin ${ }^{50}$. Moreover, it has been reported that Menin and MLL1 regulate distinct pathways during normal haematopoiesis and B-cell development ${ }^{51}$. We herein demonstrated that the primary effect of Menin deficiency was to reduce the H3K27ac, but not H3K4me3, levels at the Bach2 locus. The binding of the histone acetyltransferase, PCAF, at the Bach 2 locus was decreased in the Menin KO $\mathrm{T}_{\mathrm{H}}$ cells. These results suggest that Menin forms complexes with PCAF that have not yet been identified and preserves the Bach2 expression in CD4 T cells by maintaining H3K27 acetylation. This was further indicated by the finding that TSA treatment restored the Bach2 expression in the Menin KO $\mathrm{T}_{\mathrm{H}}$ cells. In contrast, we were unable to detect Bach2 proteins in the Menin $\mathrm{KO} \mathrm{T}_{\mathrm{H}}$ cells using immunoblotting, whereas a considerable amount of Bach2 mRNA continue to be expressed. Therefore, Menin may regulate the Bach2 expression via both transcriptional and post-transcriptional mechanism, thereby controlling $\mathrm{CD} 4 \mathrm{~T}$ senescence and cytokine homeostasis during CD4 T-cell activation.

A recent report indicated that Menin has modest effects on haematopoiesis under steady-state conditions, while playing a crucial role in the long-term maintenance of haematopoietic stem cells (HSCs), particularly under conditions of haematopoietic stress $^{52}$. A significant decrease in the number of $B$ cells was observed in the tamoxifen-administered Menin flox/flox $x$ CreER TG mice, and a mixed bone marrow transfer assay revealed a cellautonomous reduction in the generation of myeloid, $\mathrm{B}$ and $\mathrm{T}$ cells from Menin-deleted $\mathrm{HSCs}^{52}$. We found that the number of mature $\mathrm{T}$ cells was modestly decreased in the spleens of the Menin ${ }^{\text {flox/flox }} \times$ CD4-Cre TG mice. In addition, the number of Menin-deficient CD4 $T$ cells further decreased after TCR stimulation. The present study indicates that Menin maintains the number of $\mathrm{CD} 4 \mathrm{~T}$ cells in part by inhibiting cellular senescence. It is possible that Menin also plays a crucial role in inhibiting the senescence of HSCs induced by haematopoietic stress and is therefore required for the long-term maintenance of HSCs. Menin regulates haematopoiesis in part by regulating the Hox gene expression ${ }^{19}$. However, we were unable to detect a reduced Hox gene expression in the Menin $\mathrm{KO} \mathrm{T}_{\mathrm{H}}$ cells using a DNA microarray analysis. Therefore, Menin presumably regulates $\mathrm{CD} 4 \mathrm{~T}$-cell senescence and cytokine production in a Hox-independent manner.

We detected prolonged activation of NF-kB p65 (RelA) in the Menin $\mathrm{KO} \mathrm{T}_{\mathrm{H}}$ cells. The low level and prolonged activation of RelA is a common characteristic of senescent cells ${ }^{8,11}$, and RelA accumulates on the chromatin of senescent cells and acts as a master regulator of the SASP ${ }^{39}$. Menin interacts with the NF-kB components both in vitro and in vivo, and represses the transcriptional activation of an NF-kB reporter gene stimulated by phorbol-12-myristate-13-acetate or TNF- $\alpha^{24}$. Moreover, prolonged activation of RelA was also detected in the Bach2 $\mathrm{KO} \mathrm{T}_{\mathrm{H}}$ cells. Therefore, Menin modulates the NF- $\mathrm{kB}$-dependent signalling pathway in $\mathrm{CD} 4 \mathrm{~T}$ cells via multiple pathways, thereby regulating the NF-kB-mediated induction of the age-dependent gene expression and senescence.

In conclusion, we herein demonstrated that Menin controls cellular senescence and cytokine production, and it is involved in the fate decision of $\mathrm{CD} 4 \mathrm{~T}$ cells after TCR-mediated activation. Bach2 acts downstream of Menin in the regulation of the senescence and cytokine production. These findings revealed that Menin controls CD4 T-cell-mediated immune homeostasis, at least in part, by regulating the expression of Bach2. Moreover, Bach1, a homologue of Bach2, repressed the oxidative stressinduced cellular senescence of $\mathrm{MEF}^{53}$. Taken together, the data presented here indicate that Bach repressors may play a crucial role in repressing cellular senescence and in the subsequent ageassociated development of various disorders, such as cancer and inflammatory diseases.

\section{Methods}

Mice. Menin ${ }^{\text {flox/flox }}$ mice, TCR- $\beta \delta$ KO mice and Cre TG mice under the control of the $C d 4$ promoter were purchased from The Jackson Laboratory. Menin flox/flox $\times$ CD4 Cre Tg mice with BALB/c mice were generated by Dr Toshinori Nakayama (Chiba University, Chiba, Japan). Bach $2^{\text {flox/flox }}$ mice were established by Dr Tomohiro Kurosaki (RIKEN and Osaka University) ${ }^{28}$, OVA-specific $\alpha \beta T C R$ (DO11.10) TG mice were provided by Dr D. Loh (Washington University School of Medicine, St Louis, USA) ${ }^{54}$, and C57BL/6 and BALB/c mice were purchased from Clea (Clea Japan, Tokyo, Japan). The female mice were used in in vivo experiments. For in vitro experiments, both male and female mice were used. All mice were maintained under specific pathogen-free conditions and were then used at 6-10 weeks of age. All experiments using mice received approval from the Kazusa DNA Research Institute and Ehime University Administrative Panel for Animal Care. All animal care was conducted in accordance with the guidelines of the Kazusa DNA Research Institute and Ehime University.

Antibodies. Antibodies used for intracellular and cell-surface staining were as follows: anti-IL-4-phycoerythrin (PE) mAb (11B11; BD Bioscience), IFN- $\gamma$-FITC mAb (XMG1.2; BD Bioscience), IL-5-allophycocyanin (APC) (TRFK5; eBioscience), IL-13-PE (eBio13A; eBioscience), IL-2-APC mAb (JES6-5H4; eBioscience), IL-6-PE (MP5-20F3; BioLegend), IL-9-PE (RM9A4; BioLegend), IL-10-APC (JES5-16E3; BD Biosciences), IL-17A-PE (TC11-18H10.1; BioLegend), OPN-PE (cat\#IC808P; R\&D systems), Foxp3-Alexa Fluor 647 (150D; BioLegend) and NF- $\kappa B$ p65-Alexa Fluor 647 (cat\#448; Cell Signaling Technology), CD27-PE (LG.7F9; eBioscience), CD62L-APC (MEL-14; BD Biosciences), CTLA-4 (CD152) PE (UC10-4B9; eBioscience) and anti-TCR DO11.10-PE (KJ1-26; BioLegend). Antibodies for immunoblotting were as follows: anti-Menin pAb (cat\#A300-105A Bethyl Laboratories), anti-Bach2 pAb (cat\#SAB2100201; Thermo Scientific), antiNF- $\mathrm{B}$ p 65 mAb (cat\#8242; Cell Signaling Technology), anti-RelB pAb (sc-226; Santa Cruz Biotechnology) and anti- $\alpha$-tubulin mAb (cat\#MS-581-P; NeoMarkers). Antibodies used for ChIP assay were as follows: anti-histone H3K4me3 pAb (cat\#39159; Active Motif), anti-histone H3K27ac pAb (cat\#39133; Active Motif), anti-histone H3K27me3 pAb (cat\#39155; Active Motif), anti-Menin pAb (cat\#A300-105A; Bethyl Laboratories), anti-Bach2 ant-serum (cat\#SAB2100201; Thermo Scientific), anti-RNA polymerase II mAb (8WG16 cat\#05-952; MERCK MILLIPORE), anti-PCAF mAb (cat\#3378; Cell Signaling Technology), anti-MLL pAb (cat\#A300-086A; Bethyl Laboratories), anti-Rbbp5 pAb (act\#A300-109A; Bethyl Laboratories) and anti-Suz12 mAb (cat\#3737; Cell Signaling Technology). All antibodies were diluted and used according to the manufacturer's protocol.

CD4 T-cell stimulation and differentiation in vitro. Naive CD4 $\mathrm{T}$ $\left(\mathrm{CD} 44^{\text {low }} \mathrm{CD} 62 \mathrm{~L}^{\text {high }} \mathrm{CD} 25^{\text {negative }}\right)$ cells were prepared using a $\mathrm{CD} 4{ }^{+} \mathrm{CD} 62 \mathrm{~L}^{+}$ T-cell isolation kit II (Miltenyi Biotec). Naive CD4 T cells $\left(1.5 \times 10^{6}\right)$ were stimulated with immobilized anti-TCR- $\beta \mathrm{mAb}\left(3 \mu \mathrm{g} \mathrm{ml}^{-1}\right.$, H57-597, BioLegend) 
and an anti-CD28 $\mathrm{mAb}\left(1 \mu \mathrm{g} \mathrm{ml}^{-1}, 37.5\right.$, BioLegend) for 2 days under the conditions indicated. Next, cells were transferred to a new plate and cultured further in the presence of cytokines. Cytokine conditions were as follows: IL-2 $\left(\mathrm{T}_{\mathrm{H}}\right)$ conditions: IL-2 $\left(2.5 \mathrm{ng} \mathrm{ml}^{-1}\right)$; neutral conditions: IL-2 $\left(2.5 \mathrm{ng} \mathrm{ml}^{-1}\right)$, anti-IL-4 $\mathrm{mAb}\left(5 \mu \mathrm{g} \mathrm{ml}^{-1}, 11 \mathrm{~B} 11\right.$, BioLegend $)$ and anti-IFN- $\gamma \mathrm{mAb}\left(5 \mu \mathrm{g} \mathrm{ml}{ }^{-1}\right.$, R4-6A2, BioLegend).

Intracellular staining of cytokines. The cells that were differentiated in vitro were stimulated with immobilized anti-TCR- $\beta \mathrm{mAb}\left(3 \mu \mathrm{g} \mathrm{ml}{ }^{-1}, \mathrm{H} 57-597\right.$, BioLegend) for $6 \mathrm{~h}$ with monensin $(2 \mu \mathrm{M})$, and intracellular staining was performed as described. Flow cytometric analysis (FACS) was performed using a FACS Calibur (BD Biosciences), and results were analysed using FlowJo software (Tree Star)

ELISA and MILLIPLEX assay. The cells were stimulated with immobilized anti-TCR- $\beta \mathrm{mAb}$ for $16 \mathrm{~h}$. The amounts of cytokines and OPN in the supernatants were determined by ELISA, as described. The amounts of chemokines were determined using an MILLIPLEX assay kit according to the manufacturer's protocol (EMD-Millipore)

\section{Quantitative reverse transcriptase polymerase chain reaction. Total RNA} was isolated using TRIZOL Reagent, and CDNA was synthesized using the Superscript VILO cDNA synthesis kit (Life Technologies). Quantitative RT-PCR was performed as described, using Step One Plus Real-Time PCR Systems (Life Technologies). Primer sequences can be found in the Supplementary Methods.

DNA microarray analysis. The gene expression profiles of the Menin KO naive $\mathrm{CD} 4 \mathrm{~T}$ cells cultured under IL-2-conditions were analysed using the Agilent Whole Mouse $44 \mathrm{~K}$ Array. The raw data were subjected to $\log 2$ transformation and normalized using the Subio Platform (Subio).

Pull-down assay. Naive CD4 T cells were cultured under $\mathrm{T}_{\mathrm{H}}$ conditions for 7 days. Then, the cells were pelleted, resuspended in buffer C ( $20 \mathrm{mM}$ HEPES ( $\mathrm{pH} 7.9)$, $420 \mathrm{mM} \mathrm{NaCl}, 1.5 \mathrm{mM} \mathrm{MgCl}$, $0.2 \mathrm{mM}$ EDTA, $1 \mathrm{mM}$ dithiothreitol, $0.1 \%$ Nonidet P- $40,1 \mathrm{mM} \mathrm{Na}_{2} \mathrm{VO}_{3}, 1 \mathrm{mM} \mathrm{NaF}, 1 \mathrm{mM} \beta$-glycerophosphate, $1 \mathrm{mM}$ phenylmethylsulfonyl fluoride) and lysed on ice for $15 \mathrm{~min}$. Insoluble material was removed by centrifugation. The supernatant was diluted 1:3 with buffer $\mathrm{D}$ (buffer $\mathrm{C}$ without $\mathrm{NaCl})$. The lysates were incubated with $10 \mu \mathrm{g}$ of poly(dI-dC)-poly(dI-dC) (\#11-219-847, Roche) and streptavidin-agarose (\#16-126, MILLIPRE) carrying indicated biotinylated oligonucleotides for $40 \mathrm{~min}$ at $4{ }^{\circ} \mathrm{C}$. The bound proteins were eluted by adding SDS sample buffer and separated on a sodium dodecyl sulphate polyacrylamide gel and then subjected to immunoblotting with specific antibodies. Full size blots can be found in Supplementary Fig. 11. The NF- $\mathrm{KB}$ consensus oligonucleotide probes for the pull-down assay $5^{\prime}$-AGTTGAGGGGACTTTCCCA GGC- $3^{\prime}$

Retrovirus-mediated gene transfer. Retrovirus infection was performed using pMXs-IRES-hNGFR vector and a Plat-E packaging system, which is kindly, provided by Dr Toshio Kitamura (University of Tokyo, Japan). pMX-Bach2-IREShNGFR was constructed by inserting mouse Bach2 cDNA into the multi-cloning site of pMxs-IRES-hNGFR. Virus supernatant was concentrated by centrifugation $(8,000 \mathrm{~g}, 16 \mathrm{~h})$ and added to the $\mathrm{T}_{\mathrm{H}}$ cell cultures on day 2. After culturing for three more days, the infected cells were detected with anti-human NGFR-PE mAb (ME20.4-1.H4, Miltenyi Biotec). The hNGFR-positive infected cells were purified using an anti-PE microbeads (\#130-048-801, Miltenyi Biotec) and an AutoMACS device (Miltenyi Biotec)

Analysis of cell viability. The WT or Menin KO naïve CD4 T cells were cultured under IL-2-conditions for 7 days and then $10 \mu \mathrm{M}$ EdU was added for $45 \mathrm{~min}$. EdU incorporation was determined using a Clik-iT EdU Alexa Fluor 488 Flow Cytometry Assay Kit \#C35002 (Life Technologies). Cell death was detected using an FITC AnnexinV Apoptosis Detection Kit \#556547 (BD Biosciences). SA $\beta$-galactosidase assay was performed on day 10 using a Senescence $\beta$-Galactosidase Staining Kit \#9860 (Cell Signaling Technology).

ChIP assay and ChIP-seq. The Magna ChIP kit was used according to the manufacturer's protocol (EMD-Millipore). Samples for ChIP-seq were prepared according to the manufacturer's protocol (Illumina) and were sequenced using a Genome Analyzer IIx (Illumina). The obtained reads were mapped on the mouse reference genome sequence $(9 \mathrm{~mm})$ using the Bowtie 0.12 .7 software program. Peak calling was performed using the MACS 1.4.2. software program. To visualize the ChIP sequencing results, the data were converted to a wiggle file format at a 25-base resolution and uploaded to the Subio platform (Subio, Japan).

Adoptive transfer. For in vivo expansion assay of the antigen-specific CD4 T cells, CD4 T cells from DO11.10 TG mice were sorted using an AutoMACS and transplanted into BALB/c mice. Then the mice were immunized i.p. with $100 \mu \mathrm{g}$ OVA plus lipoplysaccharide $(10 \mu \mathrm{g})$. OVA-TCR ${ }^{+}$cells were detected using the $\mathrm{KJ} 1-26 \mathrm{mAb}$. To assess the survival of Menin $\mathrm{KO}_{\mathrm{H}}$ cells in vivo, the CD45.1 WT or CD45.2 Menin KO naive CD4 T cells were cultured under $\mathrm{T}_{\mathrm{H}}$ (IL-2) conditions. The WT (CD45.1) and Menin KO (CD45.2) effector CD4 T cells were mixed 1:1 $\left(5 \times 10^{6}\right.$ cells each) and transferred into the TCR- $\beta \delta$ KO mice. The ratio of CD45.1 to CD45.2 CD4 T cells was determined using FACS, at 4 weeks after transfer.

OVA-induced allergic airway inflammation. The WT and Menin KO mice were immunized i.p. with $100 \mu \mathrm{g}$ OVA in $2 \mathrm{mg}$ of aluminium hydroxide gel (day 0 ) and challenged with OVA in saline intranasally $(100 \mu \mathrm{g} /$ mouse) on days 8 and 10 . Two days later, BAL-fluid cells and lung samples were prepared for histological examination (H\&E staining).

Infection with Listeria monocytogenes. WT and Menin KO mice were infected with Listeria monocytogenes (LM) (EGD strain: $1 \times 10^{3} \mathrm{CFU}$ ) intraperitoneally (primary infection), then reinfected with LM $\left(5 \times 10^{3}\right.$ CFU) 14 days after the primary infection (secondary infection). The bacterial burden in the spleen was determined 3 days after each episode of infection.

Generation of senescent effector CD4 T cells. $\mathrm{KJ} 1^{+}$naive $\mathrm{CD} 4 \mathrm{~T}$ cells obtained from DO11.10 OVA-specific TCR TG mice were stimulated with plate bound antiTCR- $\beta$ mAb plus anti-CD28 mAb in the presence of IL- 2 for 2 days, after which the cells were further expanded with IL-2 for an additional 3 days in vitro. The cells were then transferred intravenously into syngeneic $\mathrm{BALB} / \mathrm{c} n u / n u$ recipient mice. Four weeks after the transfer, $\mathrm{KJ} 1^{+}$-transferred $\mathrm{CD} 4 \mathrm{~T}$ cells were recovered from the spleens of the recipient mice and expanded using the same method as in the primary stimulation and used as a senescent effector $\mathrm{CD} 4 \mathrm{~T}\left(\mathrm{sT}_{\mathrm{H}}\right)$ cell'.

Statistical analysis. Student's $t$-test was used for the statistical analyses.

\section{References}

1. Linton, P. J. \& Dorshkind, K. Age-related changes in lymphocyte development and function. Nat. Immunol. 5, 133-139 (2004).

2. Finkel, T., Serrano, M. \& Blasco, M. A. The common biology of cancer and ageing. Nature 448, 767-774 (2007).

3. Gavazzi, G. \& Krause, K. H. Ageing and infection. Lancet. Infect. Dis. 2, 659-666 (2002).

4. Lindstrom, T. M. \& Robinson, W. H. Rheumatoid arthritis: a role for immunosenescence? J. Am. Geriatr. Soc. 58, 1565-1575 (2010).

5. Cavanagh, M. M., Weyand, C. M. \& Goronzy, J. J. Chronic inflammation and aging: DNA damage tips the balance. Curr. Opin. Immunol. 24, 488-493 (2012).

6. Maue, A. C. et al. T-cell immunosenescence: lessons learned from mouse models of aging. Trends Immunol. 30, 301-305 (2009).

7. Haynes, L. \& Lefebvre, J. S. Age-related deficiencies in antigen-specific CD4 T cell responses: lessons from mouse models. Aging Dis. 2, 374-381 (2011).

8. Kuilman, T., Michaloglou, C., Mooi, W. J. \& Peeper, D. S. The essence of senescence. Genes Dev. 24, 2463-2479 (2010).

9. Adams, P. D. Healing and hurting: molecular mechanisms, functions, and pathologies of cellular senescence. Mol. Cell. 36, 2-14 (2009).

10. Rodier, F. \& Campisi, J. Four faces of cellular senescence. J. Cell Biol. 192, 547-556 (2011)

11. Tchkonia, T., Zhu, Y., van Deursen, J., Campisi, J. \& Kirkland, J. L. Cellular senescence and the senescent secretory phenotype: therapeutic opportunities. J. Clin. Invest. 123, 966-972 (2013).

12. Davalos, A. R., Coppe, J. P., Campisi, J. \& Desprez, P. Y. Senescent cells as a source of inflammatory factors for tumor progression. Cancer Metastasis Rev. 29, 273-283 (2010)

13. Ohtani, N. \& Hara, E. Roles and mechanisms of cellular senescence in regulation of tissue homeostasis. Cancer Sci. 104, 525-530 (2013).

14. Franceschi, C. et al. Inflamm-aging. An evolutionary perspective on immunosenescence. Ann. NY Acad. Sci. 908, 244-254 (2000).

15. Brandi, M. L. et al. Guidelines for diagnosis and therapy of MEN type 1 and type 2. J. Clin. Endocrinol. Metab. 86, 5658-5671 (2001).

16. Balogh, K., Patocs, A., Hunyady, L. \& Racz, K. Menin dynamics and functional insight: take your partners. Mol. Cell Endocrinol. 326, 80-84 (2010).

17. Yokoyama, A. et al. Leukemia proto-oncoprotein MLL forms a SET1-like histone methyltransferase complex with menin to regulate Hox gene expression. Mol. Cell Biol. 24, 5639-5649 (2004).

18. Yokoyama, A. et al. The menin tumor suppressor protein is an essential oncogenic cofactor for MLL-associated leukemogenesis. Cell 123, 207-218 (2005).

19. Chen, Y. X. et al. The tumor suppressor menin regulates hematopoiesis and myeloid transformation by influencing Hox gene expression. Proc. Natl Acad. Sci. USA 103, 1018-1023 (2006). 
20. Schuettengruber, B., Martinez, A. M., Iovino, N. \& Cavalli, G. Trithorax group proteins: switching genes on and keeping them active. Nat. Rev. Mol. Cell. Biol. 12, 799-814 (2011).

21. Hughes, C. M. et al. Menin associates with a trithorax family histone methyltransferase complex and with the hoxc8 locus. Mol. Cell. 13, 587-597 (2004).

22. Gao, S. B. et al. Suppression of lung adenocarcinoma through menin and polycomb gene-mediated repression of growth factor pleiotrophin. Oncogene 28, 4095-4104 (2009).

23. Balogh, K., Racz, K., Patocs, A. \& Hunyady, L. Menin and its interacting proteins: elucidation of menin function. Trends Endocrinol. Metab. 17, 357-364 (2006).

24. Wu, T. \& Hua, X. Menin represses tumorigenesis via repressing cell proliferation. Am. J. Cancer Res. 1, 726-739 (2011).

25. Sykiotis, G. P. \& Bohmann, D. Stress-activated cap'n'collar transcription factors in aging and human disease. Sci. Signal. 3, re3 (2010).

26. Muto, A. et al. Bach2 represses plasma cell gene regulatory network in B cells to promote antibody class switch. EMBO J. 29, 4048-4061 (2010).

27. Muto, A. et al. The transcriptional programme of antibody class switching involves the repressor Bach2. Nature 429, 566-571 (2004).

28. Kometani, K. et al. Repression of the transcription factor Bach2 contributes to predisposition of IgG1 memory B cells toward plasma cell differentiation. Immunity 39, 136-147 (2013).

29. Roychoudhuri, R. et al. $\mathrm{BACH} 2$ represses effector programs to stabilize T-mediated immune homeostasis. Nature 498, 506-510 (2013).

30. Tsukumo, S. I. et al. Bach 2 maintains $\mathrm{T}$ cells in a naive state by suppressing effector memory-related genes. Proc. Natl Acad. Sci. USA 110, 10735-10740 (2013).

31. Dubois, P. C. et al. Multiple common variants for celiac disease influencing immune gene expression. Nat. Genet. 42, 295-302 (2010).

32. Cooper, J. D. et al. Meta-analysis of genome-wide association study data identifies additional type 1 diabetes risk loci. Nat. Genet. 40, 1399-1401 (2008).

33. Christodoulou, K. et al. Next generation exome sequencing of paediatric inflammatory bowel disease patients identifies rare and novel variants in candidate genes. Gut 62, 977-984 (2013).

34. $\mathrm{Hu}, \mathrm{G}$. \& Chen, J. A genome-wide regulatory network identifies key transcription factors for memory CD8 $(+)$ T-cell development. Nat. Commun. 4, 2830 (2013).

35. Onodera, A. et al. STAT6-mediated displacement of polycomb by trithorax complex establishes long-term maintenance of GATA3 expression in T helper type 2 cells. J. Exp. Med. 207, 2493-2506 (2010).

36. Tilstra, J. S. et al. NF-kappaB inhibition delays DNA damage-induced senescence and aging in mice. J. Clin. Invest. 122, 2601-2612 (2012).

37. Adler, A. S. et al. Motif module map reveals enforcement of aging by continual NF-kappaB activity. Genes Dev. 21, 3244-3257 (2007).

38. Osorio, F. G. et al. Nuclear lamina defects cause ATM-dependent NF-kappaB activation and link accelerated aging to a systemic inflammatory response. Genes Dev. 26, 2311-2324 (2012).

39. Chien, Y. et al. Control of the senescence-associated secretory phenotype by NF-kappaB promotes senescence and enhances chemosensitivity. Genes Dev. 25, 2125-2136 (2011).

40. van Gisbergen, K. P. et al. The costimulatory molecule CD27 maintains clonally diverse CD8 $(+) \mathrm{T}$ cell responses of low antigen affinity to protect against viral variants. Immunity 35, 97-108 (2011).

41. Fearon, D. T. The expansion and maintenance of antigen-selected CD8 $(+)$ T cell clones. Adv. Immunol. 96, 103-139 (2007).

42. Moro-Garcia, M. A., Alonso-Arias, R. \& Lopez-Larrea, C. Molecular mechanisms involved in the aging of the T-cell immune response. Curr. Genomics 13, 589-602 (2012).

43. Shimatani, K., Nakashima, Y., Hattori, M., Hamazaki, Y. \& Minato, N. PD-1 + memory phenotype CD4 $+\mathrm{T}$ cells expressing C/EBPalpha underlie $\mathrm{T}$ cell immunodepression in senescence and leukemia. Proc. Natl Acad. Sci. USA 106, 15807-15812 (2009)

44. Hendel, A., Hiebert, P. R., Boivin, W. A., Williams, S. J. \& Granville, D. J. Granzymes in age-related cardiovascular and pulmonary diseases. Cell. Death Differ. 17, 596-606 (2010).
45. Freund, A., Orjalo, A. V., Desprez, P. Y. \& Campisi, J. Inflammatory networks during cellular senescence: causes and consequences. Trends Mol. Med. 16, 238-246 (2010).

46. Haynes, L. \& Swain, S. L. Aged-related shifts in T cell homeostasis lead to intrinsic T cell defects. Semin. Immunol. 24, 350-355 (2012).

47. Yokoyama, A. \& Cleary, M. L. Menin critically links MLL proteins with LEDGF on cancer-associated target genes. Cancer Cell 14, 36-46 (2008).

48. Jin, S. et al. c-Myb binds MLL through menin in human leukemia cells and is an important driver of MLL-associated leukemogenesis. J. Clin. Invest. 120, 593-606 (2010).

49. La, P. et al. Tumor suppressor menin: the essential role of nuclear localization signal domains in coordinating gene expression. Oncogene 25, 3537-3546 (2006).

50. Scacheri, P. C. et al. Genome-wide analysis of menin binding provides insights into MEN1 tumorigenesis. PLoS Genet. 2, e51 (2006).

51. Li, B. E., Gan, T., Meyerson, M., Rabbitts, T. H. \& Ernst, P. Distinct pathways regulated by menin and by MLL1 in hematopoietic stem cells and developing B cells. Blood 122, 2039-2046 (2013).

52. Maillard, I. et al. Menin regulates the function of hematopoietic stem cells and lymphoid progenitors. Blood 113, 1661-1669 (2009).

53. Dohi, Y. et al. Bach1 inhibits oxidative stress-induced cellular senescence by impeding 553 function on chromatin. Nat. Struct. Mol. Biol. 15, 1246-1254 (2008).

54. Murphy, K. M., Heimberger, A. B. \& Loh, D. Y. Induction by antigen of intrathymic apoptosis of CD4 + CD8 + TCRlo thymocytes in vivo. Science $\mathbf{2 5 0}$ 1720-1723 (1990).

\section{Acknowledgements}

We thank T. Watanabe, K. Sato, M. Takazawa, Y. Tanaka and N. Nakashio for their technical assistance. This work was supported by the JST PRESTO (Japan), JSPS KAKENHI Grant Numbers, 23390075, 25118718, 25670232, 25860376, the Kanae Foundation for the Promotion of Medical Science, the Astellas Foundation for Research on Metabolic Disorders and the Naito Foundation Natural Science Scholarship.

\section{Author contributions}

M.K. and M.Y. designed and performed the experiments, analysed the data and wrote the manuscript, J.S., S.T., M.K., S.M., A.M., O.O. and T.Y. performed the experiments K.K. and T.K. established and provided the Bach $2^{\text {flox/flox }}$ mice, T.N. provided the Menin ${ }^{\text {flox/flox }}$ mice with a BALB/c background and edited the manuscript, and M.Y. conceptualized the research, directed the study and edited the manuscript.

\section{Additional information}

Accession codes: The microarray and ChIP sequencing data were deposited in the NCBI Gene Expression Omnibus (GEO) database under accession numbers GSE49968 and GSE53831, respectively.

Supplementary Information accompanies this paper at http://www.nature.com/nature communications

Competing financial interests: The authors declare no competing financial interests.

Reprints and permission information is available online at http://npg.nature.com/ reprintsandpermissions/

How to cite this article: Kuwahara, M. et al. The Menin-Bach2 axis is critical for regulating CD4 T-cell senescence and cytokine homeostasis. Nat. Commun. 5:3555 doi: 10.1038/ncomms4555 (2014).

(c) (1) (2) This work is licensed under a Creative Commons AttributionNonCommercial-ShareAlike 3.0 Unported License. The images or other third party material in this article are included in the article's Creative Commons license, unless indicated otherwise in the credit line; if the material is not included under the Creative Commons license, users will need to obtain permission from the license holder to reproduce the material. To view a copy of this license, visit http:// creativecommons.org/licenses/by-nc-sa/3.0/ 